\title{
Fabric dependence of wave propagation in anisotropic porous media
}

\author{
Stephen C. Cowin · Luis Cardoso
}

Received: 3 January 2010 / Accepted: 8 April 2010 / Published online: 12 May 2010

(C) The Author(s) 2010. This article is published with open access at Springerlink.com

\begin{abstract}
Current diagnosis of bone loss and osteoporosis is based on the measurement of the bone mineral density (BMD) or the apparent mass density. Unfortunately, in most clinical ultrasound densitometers: 1) measurements are often performed in a single anatomical direction, 2) only the first wave arriving to the ultrasound probe is characterized, and 3 ) the analysis of bone status is based on empirical relationships between measurable quantities such as speed of sound (SOS) and broadband ultrasound attenuation (BUA) and the density of the porous medium. However, the existence of a second wave in cancellous bone has been reported, which is an unequivocal signature of poroelastic media, as predicted by Biot's poroelastic wave propagation theory. In this paper, the governing equations for wave motion in the linear theory of anisotropic poroelastic materials are developed and extended to include the dependence of the constitutive relations upon fabric - a quantitative stereological measure of the degree of structural anisotropy in the pore architecture of a porous medium. This fabric-dependent anisotropic poroelastic approach is a theoretical framework to describe the microarchitectural-dependent relationship between measurable wave properties and the elastic constants of trabecular bone, and thus represents an alternative for bone quality assessment beyond BMD alone.
\end{abstract}

Keywords Poroelasticity · Anisotropy · Incompressibility · Wave propagation $\cdot$ Bone

S. C. Cowin $(\varangle) \cdot$ L. Cardoso

The New York Center for Biomedical Engineering,

The Departments of Biomedical and Mechanical Engineering, The School of Engineering of The City College, and The Graduate School of The City University of New York, New York, NY 10031, USA

e-mail: scowin@earthlink.net

URL: http://bme.engr.ccny.cuny.edu/

\section{Introduction}

The current gold standard to diagnose bone loss and osteoporosis consists of determining the amount of bone mineral density-BMD—measured with a Dual Energy X-ray Absorptiometry (DEXA) system (Steiger 1995a,b; Formica 1998). The BMD is highly correlated to bone mass when measured in the spine, wrist and femoral neck (Steiger 1995a,b; Link et al. 2002). However, a significant number of women diagnosed with osteoporosis based on BMD measurement do not suffer fractures, whereas many women with normal BMD do (Bolotin 2007; Kleerekoper and Nelson 2005; Kaptoge et al. 2005; Bone et al. 2005; Gandolini and Salvioni 2004; Nielsen 2000). These studies have demonstrated that BMD measurements lack both sensitivity and selectivity to effectively identify patients with decreased bone strength and at risk of fracture, indicating that other factors besides bone mass play an important role in osteoporosis.

Ultrasound wave propagation is an attractive alternative to diagnose osteoporosis (Siffert and Kaufman 2006; Hans et al. 1996; Grimm and Williams 1997a) because it is nonionizing, inexpensive and non-invasive. More importantly, ultrasound waves are elastic vibrations that can provide direct information on the mechanical properties of the medium in which they propagate. Clinical ultrasound in bone is based on a wave transmission technique to measure the speed of sound (SOS) and broadband ultrasound attenuation (BUA) in the heel bone (calcaneum). Unfortunately, a major limitation associated with current clinical ultrasound systems (Grigorian et al. 2002) —often called ultrasound densitometers - consists of determining bone mass density as DEXA does, without taking advantage of the fact that ultrasound is sensitive to microarchitecture and tissue composition (Njeh et al. 2001; Nicholson et al. 1998; Sakata et al. 2004). 
In most clinical ultrasound densitometer systems, only the first wave arriving to the ultrasound probe is identified. If only one wave is measured, the analysis is limited to an "equivalent medium approach" in which the solid trabecular structure cannot be distinguished from the fluid within the pores. However, the existence of a second wave in cancellous bone has been reported (Hosokawa and Otani 1997, 1998; Cardoso et al. 2001, 2003), which is an unequivocal signature of poroelastic media. These two waves propagate with different velocities and have been shown to correspond to the fast and slow waves predicted by Biot (1941, 1955, 1956a,b, $1962 a, b)$ poroelastic wave propagation theory. Therefore, a poroelastic wave propagation theory is conceptually more appropriate than an equivalent media approach to characterize the properties of the porous medium.

The application of poroelasticity to bone tissue (Cowin 1999) and geological materials (Biot 1941, 1955, 1956a,b, 1962a,b; Plona and Johnson 1983; Sharma 2005, 2008) has been described in the past, and a number of models based on the isotropic Biot theory of wave propagation in porous media have been used (Williams 1992; Hosokawa and Otani 1997, 1998; Cardoso et al. 2008) to explain acoustic wave propagation measurements on cancellous bone. However, isotropic poroelastic models cannot explain the variability of measured wave velocities (Cardoso et al. 2003) when bone becomes anisotropic as a consequence of age and osteoporosis. Bone porosity alone is an inappropriate parameter of cancellous bone acoustic properties when the medium is anisotropic. This is because a scalar-such as porosity or any densitometry measurement-does not have the capacity to fully describe the cancellous bone architecture: a tensorial quantity such as the fabric tensor is required.

Unfortunately, most clinical ultrasound densitometers depend on empirical relationships between SOS/BUA and bone density that have failed to improve the assessment of bone loss as provided by DEXA measurements. Furthermore, measurements of SOS and BUA are performed in a single direction at the calcaneum. Such measurement cannot fully describe the properties of anisotropic bone, for which multidirectional ultrasound measurements are required. In contrast, the fabric-dependent anisotropic poroelastic approach proposed in this study has the advantage of providing a theoretical framework to describe the relationship between measurable wave properties (SOS, BUA, etc) and the elastic constants of the trabecular bone structure. Since this poroelastic wave propagation theory depends on anisotropy and tissue composition in addition to bone mass density, it represents an alternative for bone quality assessment beyond BMD.

In this paper, the governing equations for wave motion in the linear theory of anisotropic poroelastic materials are developed and extended to include the dependence of the constitutive relations upon fabric (Cowin 1985, 2004). Fabric is a quantitative stereological measure of the degree of structural anisotropy in the pore architecture of a porous medium (Hilliard 1967; Whitehouse 1974a; Whitehouse and Dyson 1974b; Oda 1976; Oda et al. 1980, 1985; Cowin and Satake 1978; Satake 1982; Kanatani 1983, 1984a,b, 1985; Harrigan and Mann 1984; Odgaard 1997a, 2001; Odgaard et al. 1997b; Matsuura et al. 2008). With the exception of the addition of the fabric variable, a tensor, the formulation of wave motions in the context of poroelastic theory is consistent with classic and contemporary literature in the field (Biot 1941, 1955, 1956a,b, 1962a,b; Plona and Johnson 1983; Sharma 2005, 2008). Unchanged by the addition of anisotropy is the fact that the total elastic volumetric response in poroelasticity is due to a combination of the elastic volumetric response of the matrix material of the porous solid, the volumetric elastic response of the pore fluid, and the pore volume changes in the porous medium. The poroelastic constitutive equations are described in the following section and, in the section after that, Biot (1956a,b, 1962a,b) formulation of wave propagation in fluid saturated porous materials is reviewed. The fabric tensor is introduced in Sect. 4, and the appropriate modification approaches of Biot (1956a,b, 1962a,b) to include fabric as a tensorial variable in the constitutive relations are described. The propagation of plane waves in an anisotropic, fabric dependent, saturated porous medium is derived in Sect. 5, and the specialization of these results to the propagation in a principal direction of fabric is presented in Sect. 6. The final section, Sect. 7, contains our discussion and concluding remarks.

\section{The poroelastic constitutive equations}

There are three sets of poroelastic constitutive equations, the stress-strain-pore pressure, the fluid content-stress-pore pressure and Darcy's law. The purpose of this section is to record the form of these constitutive equations. In the following three subsections, these equations are described in the order indicated.

\subsection{Stress-strain-pore pressure relations}

In his 1956 papers on wave propagation Biot $(1956 a, b)$ let $\mathbf{u}$ be the displacement vector of the solid matrix phase and $\mathbf{U}$ be the displacement vector of the fluid phase. These were the two basic kinematic quantities employed in those works. In Biot (1962b) the displacement vector of the fluid phase $\mathbf{U}$ was replaced by the displacement vector $\mathbf{w}$ of the fluid relative to the solid, thus

$\mathbf{w}=\mathbf{U}-\mathbf{u}$.

The present development follows Biot (1962a,b) and the two basic kinematic fields are considered to be the displacement 
vectors $\mathbf{u}$ and $\mathbf{w}$. The relative velocity of the fluid and solid components is, from (1),

$\dot{\mathbf{w}}=\dot{\mathbf{U}}-\dot{\mathbf{u}}$.

The variation in fluid content $\zeta$ is defined as

$\zeta=-\nabla \cdot \mathbf{w}$

The variation in fluid content $\zeta$ is the variation of the fluid volume per initial unit volume of the porous material due to diffusive fluid mass transport; it is defined as the difference between the volumetric strain of the pore space and the volumetric strain of the fluid volume in the pore space and is dimensionless. The remainder of the material in this section follows Cowin and Mehrabadi (2007), although the notation is slightly changed.

The relations between the strain of the porous solid and the displacement of the porous solid are written in the forms:

$$
\begin{aligned}
& \mathbf{E}=(1 / 2)\left((\nabla \otimes \mathbf{u})^{\mathrm{T}}+\nabla \otimes \mathbf{u}\right) \text { or } \\
& E_{i j}=(1 / 2)\left(u_{i, j}+u_{j, i}\right) .
\end{aligned}
$$

The stress-strain-pore pressure constitutive relation for a saturated porous medium is that the strain $E_{i j}$ in the saturated porous medium is linearly related, not only to the stress $T_{i j}$ but also to the fluid pressure $p$ in the fluid-filled pores, thus one can write the strain-stress-pore pressure relation

$E_{i j}=S_{i j k m}^{d} T_{k m}+p S_{i j k m}^{d} A_{k m}$,

or the stress-pore pressure-strain relation

$T_{i j}=C_{i j k m}^{d} E_{k m}-A_{i j} p$,

where $S_{i j k m}^{d}$ represents the drained anisotropic compliance elastic constants of the saturated porous medium and $C_{i j k m}^{d}$ is its reciprocal, the drained anisotropic elasticity tensor. They are called the drained elastic constants because they are measured in situations in which the fluid pressure $p$ in the fluidfilled pores is negligible or zero. This is achieved by draining all the pores before the test or doing the test so slowly that the pores will drain from a negligible fluid pressure. In a porous medium, the pores are assumed to be connected; there are no unconnected pores that prevent the flow of fluid through them. The three-dimensional symmetric second rank tensor $A_{i j}$, introduced by (5), is called the Biot effective stress coefficient tensor. The Biot effective stress coefficient tensor $A_{i j}$ is related to the difference between effective drained elastic constants $S_{i j k m}^{d}$ and the solid matrix material elastic compliance tensor $S_{i j k m}^{m}$ by the formula

$A_{i j}=\delta_{i j}-C_{i j k m}^{d} S_{k m q q}^{m}$.

The same for the isotropic case is represented by

$\alpha=1-K^{d} / K^{m}$.
The Biot effective stress coefficient tensor $\mathbf{A}$ is so named because it is employed in the definition of the effective stress $T_{i j}^{e f f}$ :

$T_{i j}^{e f f}=T_{i j}+A_{i j} p$.

This definition of effective stress reduces the stressstrain-pressure relation (5) to the same form as Hooke's law, thus

$E_{i j}=S_{i j k m}^{d} T_{k m}^{e f f}$.

The advantage of the representation (10) is that the fluid-saturated porous material may be thought of as an ordinary elastic material with a compliance matrix $S_{i j k m}^{d}$, but one subjected to the "effective stress" $T_{i j}^{e f f}$ rather than an (ordinary) stress $T_{i j}$. The drained elastic compliance tensor $S_{i j k m}^{d}$ may be evaluated from knowledge of the pore structure of the medium and the matrix elastic compliance tensor $S_{i j k m}^{m}$, or vice versa, using composite or effective medium theory. This constitutive equation is a modification of Hooke's law to include the effect of the pore pressure. When $p=0$ the stress-strain-pore pressure relations (5) and (6) or (10) coincide with anisotropic Hooke's law.

The relationship between a compliance tensor $S_{i j k m}$ (either $S_{i j k m}^{m}$ or $S_{i j k m}^{d}$ ) and the effective bulk modulus is simple and important in these developments. In order to develop this relationship, we begin by noting that $S_{p p q q}$, where $S_{i j k m}$ is the isotropic elastic compliance tensor, is equal to $3(1-2 v) E^{-1}$, which, in turn, is the reciprocal of the isotropic bulk modulus $K$,

$S_{p p q q}=\frac{3(1-2 v)}{E} \equiv \frac{1}{K}$.

Hill (1952) showed that $K_{\text {Reff }}=\left(S_{p p q q}\right)^{-1}$, where $S_{i j k m}$ is now the anisotropic elastic compliance tensor, is the Reuss lower bound on the effective (isotropic) bulk modulus of the anisotropic elastic material $S_{i j k m}$ and that the Voigt effective bulk modulus of an anisotropic elastic material, $K_{V e f f}=$ $\left(C_{p p q q}\right) / 9$, is the upper bound, $K_{R e f f} \leq K_{\text {eff }} \leq K_{V e f f}$. In the case of isotropy, the two bounds coincide with the isotropic bulk modulus, $K$, thus:

$\frac{1}{K_{\text {Reff }}}=\frac{1}{K_{\text {eff }}}=\frac{1}{K_{V e f f}}=S_{p p q q}=\frac{3(1-2 v)}{E} \equiv \frac{1}{K}$

where $E$ is the isotropic Young's modulus and $v$ is the Poisson's ratio. The Reuss effective bulk modulus of an anisotropic elastic material $K_{\text {Reff }}$ occurs naturally in anisotropic poroelastic theory as shown, but not noted, by Thompson and Willis (1991). Thus, using a general form of (7) for 
the orthotropic elastic compliance tensor $S_{i j k m}^{i}, i=$ mord, the following two definitions are introduced:

$$
\begin{aligned}
\frac{1}{K_{\text {Reff }}^{i}}=S_{p p q q}^{i}= & \frac{1}{E_{1}^{i}}+\frac{1}{E_{2}^{i}}+\frac{1}{E_{3}^{i}}-\frac{2 \nu_{23}^{i}}{E_{2}^{i}}-\frac{2 v_{31}^{i}}{E_{3}^{i}} \\
& -\frac{2 \nu_{12}^{i}}{E_{1}^{i}}, \quad i=m \text { or } d .
\end{aligned}
$$

\subsection{Fluid content-stress-pore pressure relations}

The fluid content-stress-pore pressure constitutive relation involves all the basic field variables for poroelasticity, the total stress $T_{i j}$, the pore pressure $p$, the strain in the solid matrix $E_{i j}$, and the variation in (dimensionless) fluid content $\zeta$. The variation in fluid content $\zeta$ depends not only upon the strain $E_{i j}$ in the solid matrix but also upon the strain induced by the pore pressure $p$, thus

$\zeta=A_{i j} E_{i j}+\Lambda p$

where

$\Lambda=S_{p p i j}^{m} A_{i j}+\phi\left(\frac{1}{K^{f}}-\frac{1}{K_{R e f f}^{m}}\right)$,

and where $\phi$ is the porosity, $K^{f}$ is the bulk modulus of the fluid and $K_{\text {Reff }}^{m}$ is defined by (13). The first term of Eq. (14) is the volumetric strain of the pore space and the second term is the volumetric strain of the fluid volume. This can be shown along the lines of reasoning used by Biot and Willis for isotropic media. The isotropic equivalent of (14) is

$\zeta=\alpha \operatorname{tr}(\varepsilon)+M p$

In the case of isotropy, this expression for $\Lambda$ reduces to

$\Lambda=\frac{1}{K^{m}}\left(1-\frac{K^{d}}{K^{m}}\right)+\phi\left(\frac{1}{K^{f}}-\frac{1}{K^{m}}\right)$.

The variation in fluid content $\zeta$ is also linearly related to both the stress $T_{i j}$, and the pore pressure $p$,

$\zeta=A_{i j} S_{i j k m}^{d} T_{k m}+C_{e f f}^{d} p$

where

$C_{e f f}^{d}=\frac{1}{K_{\text {Reff }}^{d}}-\frac{1}{K_{\text {Reff }}^{m}}+\phi\left(\frac{1}{K^{f}}-\frac{1}{K_{\text {Reff }}^{m}}\right)$.

The relation between $C_{e f f}^{d}$ and $\Lambda$ is

$C_{e f f}^{d}=\Lambda-S_{p p i j}^{m} A_{i j}+\left(\frac{1}{K_{\text {Reff }}^{d}}-\frac{1}{K_{\text {Reff }}^{m}}\right)$.

The inverse of (18) is

$p=M\left(\zeta-A_{i j} E_{i j}\right), \quad M=\frac{1}{C_{e f f}^{d}-A_{i j} S_{i j k m}^{d} A_{k m}}$, and the representation of stress $T_{i j}$ as a function of the strain $E_{i j}$ and the variation in fluid content $\zeta$, rather than as a function of the strain and the pore pressure $p$, is

$T_{i j}=\left(C_{i j k m}^{d}+M A_{k m} A_{i j}\right) E_{k m}-M A_{i j} \zeta$.

Biot (1962a,b) employed a slightly different notation for the two previous expressions, namely

$p=M_{k m} E_{k m}+M \zeta$

and

$T_{i j}=A_{i j k m} E_{k m}-M_{i j} \zeta$

where Biot's parameters $M, M_{i j}$ and $A_{i j k m}$ are related to $C_{e f f}^{d}, A_{i j}$ and $C_{i j k m}^{d}$ above by

$M_{i j}=M A_{i j}, A_{i j k m}=C_{i j k m}^{d}+M A_{i j} A_{k m}$.

Please note that our definition of $M_{i j}$ differs from that of Biot (1962b), being of the opposite sign. It is interesting to note that Biot's elasticity tensor $A_{i j \mathrm{~km}}$ differs from the drained elasticity tensor $C_{i j k m}^{d}$ by the term $M A_{i j} A_{k m}$, which is the open product of the Biot effective stress coefficient tensor $\mathbf{A}$ with itself.

\subsection{Darcy's law}

The constitutive equations of poroelasticity developed thus far are the strain-stress-pore pressure relations (6) and the fluid content-stress-pore pressure relation (14). We follow Biot (1962a,b) here and employ the equivalent relations, replacing the pore pressure by the variation in fluid content, (23) and (24). The other constitutive relation of poroelasticity is Darcy's law, relating the fluid mass flow rate, $\rho_{f} \mathbf{v}$, to the gradient $(\nabla p)$ of the pore pressure $p$ (Darcy 1856),

$\rho_{f} v_{i}=-H_{i j} \frac{\partial p}{\partial x_{j}}, H_{i j}=H_{j i}$

This relation is true if the fluid in the porespace is Newtonian; however, Darcy's law (26) applies with reasonable accuracy to many types of non-Newtonian conditions as well.

The coefficient tensor $H_{i j}$ in Darcy's law may be represented by $H_{i j}=\rho_{f_{o}} K_{i j} / \mu$ where $K_{i j}$ is the intrinsic Darcy's law permeability tensor, $\rho_{f o}$ is a reference value of the fluid density and $\mu$ is the fluid viscosity. The intrinsic permeability tensor $K_{i j}$ has units of length squared and is a function of the porous structure only, not the fluid in the pores; thus, Darcy's law (26) takes the form

$q_{i}=\phi\left(\frac{\rho_{f}}{\rho_{f_{o}}}\right) v_{i}=-\frac{K_{i j}}{\mu} \frac{\partial p}{\partial x_{j}}, K_{i j}=K_{j i}$,

where the mass flux $q_{i}$ has the dimensions of velocity. 


\section{Biot's formulation of the two-constituent momentum conservation}

Biot (1956a,b, 1962a,b) employs a Lagrangian formulation of the momentum conservation equations for the solid and the fluid phases to provide a method of representing the inertial interaction between the two phases. The Lagrangian formulation relates the kinetic energy $T$ and the viscous dissipation $D$ to the basic kinematic fields $\mathbf{v}$ and $\mathbf{w}$ by (Biot $1962 \mathrm{~b}$, equation 8.23)

$T_{i j, j}=\frac{d}{d t}\left(\frac{\partial T}{\partial \dot{u}_{i}}\right),-\frac{\partial p}{\partial x_{i}}=\frac{d}{d t}\left(\frac{\partial T}{\partial \dot{w}_{i}}\right)+\frac{\partial D}{\partial \dot{w}_{i}}$

where the kinetic energy and the viscous dissipation are assumed to be of the following forms (Biot 1962b, equations $8.16,4.16)$ :

$$
\begin{aligned}
T & =\frac{1}{2} \rho \dot{u}_{k} \dot{u}_{k}+\rho_{f} \dot{w}_{k} \dot{u}_{k}+\frac{1}{2} \rho_{f} \dot{w}_{k} J_{k m} \dot{w}_{m}, \\
\rho & =(1-\phi) \rho_{s}+\phi \rho_{f}, \\
D & =\frac{1}{2} \rho_{f} \mu \dot{w}_{k} R_{k m} \dot{w}_{m}, R_{k m}=R_{m k}, R_{k m}=K_{k m}^{-1} .
\end{aligned}
$$

In the two equations above $T, D, K_{i j}, \dot{\mathbf{u}}, \dot{\mathbf{w}}, \rho_{f}, \phi$, and $\mu$ have been defined earlier, the bulk density $\rho$ and the flowresistivity tensor $R_{k m}$ are defined in the equations above, and $\rho_{s}$ and $J_{k m}$ will now be defined. $\rho_{s}$ represents the density of the solid matrix material and $J_{k m}$ is the micro-macro velocity average tensor; it functions like a density distribution function that relates the relative micro-solid-fluid velocity to its bulk volume average $\dot{\mathbf{w}}$.

The field equations of motion are obtained by substituting (29) and (30) into (28) and then employing the constitutive Eqs. (23) and (24) and the relationship (3), $\zeta=-\nabla \cdot \mathbf{w}$, between the variation in fluid content $\zeta$ and the divergence of the displacement vector $\mathbf{w}$ of the fluid relative to the solid, thus (Biot 1962b; Sharma 2005)

$$
\begin{aligned}
& A_{i j k m} \frac{\partial^{2} u_{k}}{\partial x_{m} \partial x_{j}}+M_{i j} \frac{\partial^{2} w_{k}}{\partial x_{k} \partial x_{j}}=\rho \ddot{u}_{i}+\rho_{f} \ddot{w}_{i}, \\
& M_{k m} \frac{\partial^{2} u_{k}}{\partial x_{m} \partial x_{i}}+M \frac{\partial^{2} w_{k}}{\partial x_{k} \partial x_{i}}=\rho_{f}\left(\ddot{u}_{i}+J_{i j} \ddot{w}_{j}+\mu R_{i j} \dot{w}_{j}\right) .
\end{aligned}
$$

Equations (31) and (32) are two coupled wave equations for the solid displacement field $\mathbf{u}$ and the displacement field $\mathbf{w}$ of the fluid relative to the solid.

\section{The fabric tensor}

The fabric tensor employed is a quantitative stereological measure of the degree of structural anisotropy in the pore architecture of a porous medium. It is shown that the undrained elastic constants may be expressed as functions of the fabric tensor, the drained elastic constants, the porosity, the bulk moduli of the fluid and the matrix material.

The experimental procedure for the surface area orientation measurement of cancellous bone is described by Whitehouse (1974a), Whitehouse and Dyson (1974b), Harrigan and Mann (1984) and Turner and Cowin (1987), Turner et al. (1990). The work of these authors, and Odgaard (1997a), Odgaard (2001), Odgaard et al. (1997b), Van Rietbergen et al. (1996), Van Rietbergen et al. (1998), Yang et al. (1999), Matsuura et al. (2008) and others, has shown that the fabric tensor is a good measure of the structural anisotropy in cancellous bone tissue (Cowin 1997). The methodology of making measurements is easily adapted to an automated computational system as shown by Harrigan and Mann (1984) and Turner et al. (1990).

F denotes the fabric tensor, which is dimensionless. It is symmetric, $\mathbf{F}=\mathbf{F}^{T}$, and, as with any second rank tensor, the invariants $I, I I$ and $I I I$ are related to the traces of $\mathbf{F}, \mathbf{F}^{2}$ and $\mathbf{F}^{3}$ by the formulas recorded, for example, in Ericksen (1960):

$I=\operatorname{tr} \mathbf{F}, I I=\frac{1}{2}\left\{(\operatorname{tr} \mathbf{F})^{2}-t r \mathbf{F}^{2}\right\}$,
$I I I=\frac{1}{6}\left\{t r \mathbf{F}-3 t r \mathbf{F}^{2}+2 t r \mathbf{F}^{3}\right\}$.

The fact that a matrix satisfies its own characteristic equation, the Cayley-Hamilton theorem, is then written in the form:

$\mathbf{F}^{3}-I \mathbf{F}^{2}+I I \mathbf{F}-I I I \mathbf{1}=0$.

The significance of this result is that any power of $\mathbf{F}$ of the order three or higher may be eliminated by repetitive use of this result. From the first and second equations of (33), one can see that $\operatorname{tr} \mathbf{F}^{2}=I^{2}-2 I I$. Using the Cayley-Hamilton theorem, it is easy to show that

$\operatorname{tr} \mathbf{F}^{3}=I^{3}-3 I \cdot I I+3 I I I$ and

$\operatorname{tr} \mathbf{F}^{4}=I^{4}-3 I^{2} \cdot I I+2 I I^{2}+4 I \cdot I I I ;$

these results will be used in the following paragraphs. Finally, we normalize the fabric tensor by setting $I=\operatorname{tr} \mathbf{F}=1$. Thus, in the applications of the formulas $\operatorname{tr} \mathbf{F}^{2}=I^{2}-2 I I$ and (35), $I$ is replaced by 1 .

The relationship between the fourth rank tensor of elastic constants of a porous, anisotropic, linear elastic material and stereological parameters characterizing the anisotropy of the microstructure of the material was presented by Cowin (1985). If it is assumed that the fourth-rank elastic compliance tensor is a function only of the fabric tensor and some scalar parameters, then, using tensor algebra, it can be shown, 
Cowin (1985), that the relationship between the fourth-rank elastic compliance tensor and the fabric tensor is

$$
\begin{aligned}
S_{i j k m}= & a_{1} \delta_{i j} \delta_{k m}+a_{2}\left(F_{i j} \delta_{k m}+\delta_{i j} F_{k m}\right) \\
& +a_{3}\left(\delta_{i j} F_{k q} F_{q m}+\delta_{k m} F_{i q} F_{q j}\right) \\
& +b_{1} F_{i j} F_{k m}+b_{2}\left(F_{i j} F_{k q} F_{q m}+F_{k m} F_{i q} F_{q j}\right) \\
& +b_{3} F_{i s} F_{s j} F_{k q} F_{q m}+c_{1}\left(\delta_{k i} \delta_{m j}+\delta_{m i} \delta_{k j}\right) \\
& +c_{2}\left(F_{k i} \delta_{m j}+F_{k j} \delta_{m i}+F_{i m} \delta_{k j}+F_{m j} \delta_{k i}\right) \\
& +c_{3}\left(F_{i r} F_{r k} \delta_{m j}+F_{k r} F_{r j} \delta_{m i}+F_{i r} F_{r m} \delta_{k j}\right. \\
& \left.+F_{m r} F_{r j} \delta_{i k}\right)
\end{aligned}
$$

where $a_{1}, a_{2}, a_{3}, b_{1}, b_{2}, b_{3}, c_{1}, c_{2}$ and $c_{3}$ are functions of $\phi$ and the two invariants of $\mathbf{F}$, II and III given by (33), and where $\phi$ is the porosity or solid volume fraction of the material. The least elastic material symmetry for which the representation holds is orthotropy. It therefore holds for transverse isotropy and isotropy as well as orthotropy. The symmetry of the material is orthotropic if the three eigenvalues of $\mathbf{F}$ are distinct, transverse isotropy if only two are distinct and isotropy if all three eigenvalues are equal.

As noted earlier, the result (36) is based on the assumption that the matrix material is isotropic and that the anisotropy of the solid porous material is determined by the fabric tensor. In the case of cancellous bone, the validity of that assumption has been experimentally verified by the work of Odgaard et al. (1997b). These authors concluded that the elastic and fabric main directions coincide in cancellous bone; cancellous bone is an elastic, highly porous material. Odgaard et al. (1997b) make the following remark: "Cowin's fabric mechanics relations (Cowin 1985, 1986) (Eq. 36) implicitly assume mechanical and fabric main directions must be aligned. This may seem an intuitively acceptable assumption, but experiment support has not previously been given." The fact that the mechanical and fabric main directions coincide in (36) is a direct algebraic consequence of the constitutive assumption that the stress is an isotropic function of the strain tensor and the normalized fabric tensor only. An explicit meaning of this assumption is that only architectural features measured by the normalized fabric tensor measure, and not by any other features determine the mechanical anisotropy of the material. This same assumption is explicit in the related computation work of Van Rietbergen et al. (1996), and thus in Odgaard et al. (1997b). The experimental result of Odgaard et al. (1997b) is thus a possible justification for the assumption of isotropy for the matrix material. This does not mean that the matrix material is isotropic, it only means that there is little or no error in assuming that it is isotropic in the relationship (36).

The dependence of the drained compliance tensor upon fabric is, from (36),

$$
\begin{aligned}
S_{i j k m}^{d}= & a_{1}^{s d} \delta_{i j} \delta_{k m}+a_{2}^{s d}\left(F_{i j} \delta_{k m}+\delta_{i j} F_{k m}\right) \\
& +a_{3}^{s d}\left(\delta_{i j} F_{k q} F_{q m}+\delta_{k m} F_{i q} F_{q j}\right) \\
& +b_{1}^{s d} F_{i j} F_{k m}+b_{2}^{s d}\left(F_{i j} F_{k q} F_{q m}+F_{k m} F_{i q} F_{q j}\right) \\
& +b_{3}^{s d} F_{i s} F_{s j} F_{k q} F_{q m}+c_{1}^{s d}\left(\delta_{k i} \delta_{m j}+\delta_{m i} \delta_{k j}\right) \\
& +c_{2}^{s d}\left(F_{k i} \delta_{m j}+F_{k j} \delta_{m i}+F_{i m} \delta_{k j}+F_{m j} \delta_{k i}\right) \\
& +c_{3}^{s d}\left(F_{i r} F_{r k} \delta_{m j}+F_{k r} F_{r j} \delta_{m i}+F_{i r} F_{r m} \delta_{k j}\right. \\
& \left.+F_{m r} F_{r j} \delta_{i k}\right) .
\end{aligned}
$$

The three Young's moduli and the three shear moduli are expressed in terms of the porosity $\phi$ and the principal values of the fabric $F_{i}, i=1,2,3$ (see appendix).

$$
\begin{aligned}
\frac{1}{E_{i}}= & \frac{1}{E_{s}}\left[k_{1}^{s d}+2 k_{6}^{s d}+\left(k_{2}^{s d}+2 k_{7}^{s d}\right) I I\right. \\
& \left.+2\left(k_{3}^{s d}+2 k_{8}^{s d}\right) F_{i}+\left(2 k_{4}^{s d}+k_{5}^{s d}+4 k_{9}^{s d}\right) F_{i}^{2}\right]
\end{aligned}
$$

and

$\frac{1}{G_{i j}}=\frac{1}{G_{s}}\left[k_{6}^{s d}+k_{7}^{s d} I I+k_{8}^{s d}\left(F_{i}+F_{j}\right)+k_{9}^{s d}\left(F_{i}^{2}+F_{j}^{2}\right)\right]$

where the coefficients $k_{1}^{s d}$ through $k_{9}^{s d}$ were originally expressed (Turner et al. 1990) in terms of the apparent or bulk density, which is equal to $(1-\phi)$ times the solid bone matrix density, and here redefined in the appendix. The expressions above specify the fabric and porosity dependence of six of the nine distinct elastic constants of orthotropic symmetry; the missing three are those that involve Poisson's ratios, $\frac{\nu_{12}}{E_{1}}=\frac{\nu_{21}}{E_{2}}, \frac{\nu_{13}}{E_{1}}=\frac{\nu_{31}}{E_{3}}$ and $\frac{\nu_{32}}{E_{3}}=\frac{\nu_{23}}{E_{2}}$, which are then determined from:

$$
\begin{aligned}
v_{i j}= & -E_{i}\left[k_{1}^{s d}+k_{2}^{s d} I I+k_{3}^{s d}\left(F_{i}+F_{j}\right)\right. \\
& \left.+k_{4}^{s d}\left(F_{i}^{2}+F_{j}^{2}\right)+k_{5}^{s d} F_{i} F_{j}\right] .
\end{aligned}
$$

The form of the functional dependence of the drained elasticity tensor upon fabric is the same as compliance given by (37), but with different scalar valued functions of $\phi, I I$ and III, thus

$$
\begin{aligned}
C_{i j k m}^{d}= & a_{1}^{c d} \delta_{i j} \delta_{k m}+a_{2}^{c d}\left(F_{i j} \delta_{k m}+\delta_{i j} F_{k m}\right) \\
& +a_{3}^{c d}\left(\delta_{i j} F_{k q} F_{q m}+\delta_{k m} F_{i q} F_{q j}\right) \\
& +b_{1}^{c d} F_{i j} F_{k m}+b_{2}^{c d}\left(F_{i j} F_{k q} F_{q m}+F_{k m} F_{i q} F_{q j}\right) \\
& +b_{3}^{c d} F_{i s} F_{s j} F_{k q} F_{q m}+c_{1}^{c d}\left(\delta_{k i} \delta_{m j}+\delta_{m i} \delta_{k j}\right) \\
& +c_{2}^{c d}\left(F_{k i} \delta_{m j}+F_{k j} \delta_{m i}+F_{i m} \delta_{k j}+F_{m j} \delta_{k i}\right) \\
& +c_{3}^{c d}\left(F_{i r} F_{r k} \delta_{m j}+F_{k r} F_{r j} \delta_{m i}+F_{i r} F_{r m} \delta_{k j}\right. \\
& \left.+F_{m r} F_{r j} \delta_{i k}\right) .
\end{aligned}
$$


Recall that the Biot effective stress coefficient tensor $A_{i j}$ is related to the difference between effective drained elastic constants $C_{i j k m}^{d}$ and the solid matrix material elastic compliance tensor $S_{i j k m}^{m}$ by the formula (7), where $C_{i j k m}^{d}$ is expressed in terms of the fabric tensor by (41) above and $S_{i j k m}^{m}$ is not a function of the fabric tensor because it represents the elastic constants of the matrix material. Recall that the result (37) was based on the assumption that the matrix material is isotropic and that the anisotropy of the solid porous material is determined by the fabric tensor; thus, we express $S_{i j k m}^{m}$ in terms of the bulk modulus and the shear modulus, $K^{m}$ and $G$, respectively:

$S_{i j k m}^{m}=\frac{1}{2 G}\left(\delta_{i k} \delta_{j m}-\frac{1}{3} \delta_{i j} \delta_{k m}\right)+\frac{1}{9 K^{m}} \delta_{i j} \delta_{k m}$.

The form of $S_{i j k m}^{m}$ that appears in (7) is $S_{k m q q}^{m}$ and it is given by (42) as

$S_{k m q q}^{m}=\frac{1}{3 K^{m}} \delta_{k m}$.

Substituting (42) and (37) into (7) and simplifying, one finds that the Biot effective stress coefficient tensor $A_{i j}$ is related to the fabric tensor $\mathbf{F}$ by

$A_{i j}=\delta_{i j}-\frac{1}{3 K^{m}}\left\{a_{o}^{c d} \delta_{i j}+a_{I}^{c d} F_{i j}+a_{I I}^{c d} F_{i q} F_{q j}\right\}$

where

$a_{o}^{c d}=3 a_{1}^{c d}+a_{2}^{c d}+a_{3}^{c d}(1-2 I I)+2 c_{1}^{c d}$,
$a_{I}^{c d}=3 a_{2}^{c d}+b_{1}^{c d}+b_{2}^{c d}(1-2 I I)+4 c_{2}^{c d}$
$a_{I I}^{c d}=3 a_{3}^{c d}+b_{2}^{c d}+b_{3}^{c d}(1-2 I I)+4 c_{3}^{c d}$.

The open product of $A_{i j}$ with itself, an expression that occurs in the formula relating $A_{i j k m}$ and $C_{i j k m}^{d}$, is from (43)

$$
\begin{aligned}
A_{i j} A_{k m}= & \left\{\left(1-\frac{a_{o}^{c d}}{3 K^{m}}\right)^{2} \delta_{i j} \delta_{k m}\right. \\
& -\left(1-\frac{a_{o}^{c d}}{3 K^{m}}\right) \frac{a_{I}^{c d}}{3 K^{m}}\left(\delta_{i j} F_{k m}+F_{i j} \delta_{k m}\right) \\
& -\left(1-\frac{a_{o}^{c d}}{3 K^{m}}\right) \frac{a_{I I}^{c d}}{3 K^{m}}\left(\delta_{i j} F_{k q} F_{q m}+F_{i q} F_{q j} \delta_{k m}\right) \\
& +\left(\frac{a_{I}^{c d}}{3 K^{m}}\right)^{2} F_{i j} F_{k m}+\frac{a_{I}^{c d} a_{I I}^{c d}}{\left(3 K^{m}\right)^{2}}\left(F_{i j} F_{k q} F_{q m}\right. \\
4 p t] \quad & \left.F_{i q} F_{q j} F_{k m}\right)+\left(\frac{a_{I I}^{c d}}{3 K^{m}}\right)^{2} F_{i q} F_{q j} F_{k q} F_{q m} .
\end{aligned}
$$

Complete contraction of (46) yields $\operatorname{tr} \mathbf{A}^{2}$, a quantity that appears in all the equations of (24) for Biot's parameters
$M, M_{i j}$ and $A_{i j k m}$,

$$
\begin{aligned}
\operatorname{tr} \mathbf{A}^{2}= & 3\left(1-\frac{a_{o}^{c d}}{3 K^{m}}\right)^{2}-2\left(1-\frac{a_{o}^{c d}}{3 K^{m}}\right) a_{I}^{d} I \\
& -\left\{2 \frac{a_{I I}^{c d}}{3 K^{m}}-2 \frac{a_{o}^{c d} a_{I I}^{c d}}{\left(3 K^{m}\right)^{2}}+\left(\frac{a_{I}^{c d}}{3 K^{m}}\right)^{2}\right\}(1-2 I I) \\
& +\frac{a_{I}^{c d} a_{I I}^{c d}}{\left(3 K^{m}\right)^{2}}(1-3 I I+3 I I I) \\
& +\left(\frac{a_{I I}^{c d}}{3 K^{m}}\right)^{2}\left(1-3 I I+2 I I^{2}+4 I I I\right)
\end{aligned}
$$

Biot's parameters $M_{i j}$ and $A_{i j k m}$ are related to $C_{e f f}^{d}, A_{i j}$ and $C_{i j k m}^{d}$ above by Eq. (24). Formulas relating $M_{i j}$ and $A_{i j k m}$ directly to the fabric tensor $\mathbf{F}$ will now be obtained by using the formula (40) above expressing $C_{i j k m}^{d}$ in terms of the fabric tensor and the expression (44) relating $A_{i j}$ to fabric, thus

$M_{i j}=M \delta_{i j}-\frac{M}{3 K^{m}}\left\{a_{o}^{c d} \delta_{i j}+a_{I}^{c d} F_{i j}+a_{I I}^{c d} F_{i q} F_{q j}\right\}$,

and

$$
\begin{aligned}
A_{i j k m}= & \left(a_{1}^{c d}+M \frac{\left(3 K^{m}-a_{o}^{d}\right)^{2}}{\left(3 K^{m}\right)^{2}}\right) \delta_{i j} \delta_{k m} \\
& +\left(a_{2}^{c d}-\frac{M\left(3 K^{m}-a_{o}^{d}\right) a_{I}^{d}}{\left(3 K^{m}\right)^{2}}\right)\left(F_{i j} \delta_{k m}+\delta_{i j} F_{k m}\right) \\
& +\left(a_{3}^{c d}-M \frac{\left(3 K^{m}-a_{o}^{c d}\right) a_{I I}^{c d}}{\left(3 K^{m}\right)^{2}}\right)\left(\delta_{i j} F_{k q} F_{q m}\right. \\
& \left.+\delta_{k m} F_{i q} F_{q j}\right)+\left(b_{1}^{c d}+M \frac{\left(a_{I}^{c d}\right)^{2}}{\left(3 K^{m}\right)^{2}}\right) F_{i j} F_{k m} \\
& +\left(b_{2}^{c d}+M \frac{a_{I}^{c d} a_{I I}^{c d}}{\left(3 K^{m}\right)^{2}}\right)\left(F_{i j} F_{k q} F_{q m}+F_{k m} F_{i q} F_{q j}\right) \\
& +\left(b_{3}^{c d}+M \frac{\left(a_{I I}^{c d}\right)^{2}}{\left(3 K^{m}\right)^{2}}\right) F_{i s} F_{s j} F_{k q} F_{q m} \\
& +c_{1}^{c d}\left(\delta_{k i} \delta_{m j}+\delta_{m i} \delta_{k j}\right) \\
& +c_{2}^{c d}\left(F_{k i} \delta_{m j}+F_{k j} \delta_{m i}+F_{i m} \delta_{k j}+F_{m j} \delta_{k i}\right) \\
& +c_{3}^{c d}\left(F_{i r} F_{r k} \delta_{m j}+F_{k r} F_{r j} \delta_{m i}+F_{i r} F_{r m} \delta_{k j}\right. \\
& \left.+F_{m r} F_{r j} \delta_{i k}\right) .
\end{aligned}
$$




\section{Wave propagation in anisotropic porous media}

The propagation of a plane wave is represented kinematically by a direction of propagation, denoted by $\mathbf{n}$, a unit normal to the wave front, and $\mathbf{a}$ orb, which are the directions of displacement for the wave fronts associated with $\mathbf{u}$ and $\mathbf{w}$, respectively. These two plane waves are represented by

$$
\begin{aligned}
\mathbf{u}(\mathbf{x}, t) & =\mathbf{a} e^{i \omega\left(\frac{\mathbf{n} \cdot \mathbf{x}}{v}-t\right)}=\mathbf{a} e^{i((k+i \alpha) \mathbf{n} \cdot \mathbf{x}-\omega t)} \\
& =\mathbf{a} e^{-\alpha \mathbf{n} \cdot \mathbf{x}} e^{i(k \mathbf{n} \cdot \mathbf{x}-\omega t)} \\
w(\mathbf{x}, t) & =\mathbf{b} e^{i \omega\left(\frac{\mathbf{n} \cdot \mathbf{x}}{v}-t\right)}=\mathbf{b} e^{i((k+i \alpha) \mathbf{n} \cdot \mathbf{x}-\omega t)} \\
& =\mathbf{b} e^{-\alpha \mathbf{n} \cdot \mathbf{x}} e^{i(k \mathbf{n} \cdot \mathbf{x}-\omega t)}
\end{aligned}
$$

where $v$ is the wave phase velocity in the direction $\mathbf{n}, \mathbf{x}$ is the position vector, $\omega$ is the frequency, and $t$ is time. The relationship between the phase velocity $v$ and frequency $\omega$ of attenuating waves is a complex quantity, here represented by $k_{R e}+i \alpha_{I m}$,

$v=\frac{\omega}{k_{R e}+i \alpha_{I m}}$.

The imaginary part $\alpha$ is related to the wave attenuation as a function of traveled distance $\left(e^{-\alpha \mathbf{n} \cdot \mathbf{x}}\right)$ and the real part $k$ describes the wave vector and points in the direction $\mathbf{n}$. A transverse wave is characterized by $\mathbf{a} \cdot \mathbf{n}=0$, a longitudinal wave by $\mathbf{a} \cdot \mathbf{n}=1$. Substituting the representations (50) for the plane waves into the field Eqs. (31) and (32) one obtains equations that are in Biot $(1962 a, b)$ and Sharma (2005, 2008) and many other places,

$$
\begin{aligned}
& \left(Q_{i k}-\rho v^{2} \delta_{i k}\right) a_{k}+\left(C_{i k}-\rho_{f} v^{2} \delta_{i k}\right) b_{k}=0, \\
& \left(Q_{i k}-\rho v^{2} \delta_{i k}\right) a_{k}+\left(C_{i k}-\rho_{f} v^{2} \delta_{i k}\right) b_{k}=0,
\end{aligned}
$$

where the following notation has been introduced:

$$
Q_{i k}=A_{i j k m} n_{m} n_{j}, C_{i k}=M_{i j} n_{j} n_{k}
$$

$\mathbf{Q}$ is the acoustical tensor from elastic wave propagation and $\mathbf{C}$ represents the interaction of the velocity fields' $\mathbf{u}$ and w. Rewritten in matrix notation Eqs. (53) and (54) take the form

$$
\begin{aligned}
& \left(\mathbf{Q}-\rho v^{2} \mathbf{1}\right) \cdot \mathbf{a}+\left(\mathbf{C}-\rho_{f} v^{2} \mathbf{1}\right) \cdot \mathbf{b}=0 \\
& \left(\mathbf{C}-\rho_{f} v^{2} \mathbf{1}\right) \cdot \mathbf{a}+\left(M \mathbf{n} \otimes \mathbf{n}-\rho_{f} v^{2}\left\{\mathbf{J}+\frac{i \mu}{\omega} \mathbf{R}\right\}\right) \cdot \mathbf{b}=0
\end{aligned}
$$

Equations (55) and (56) represent an eigenvalues problem, the squares of the wave speeds $v^{2}$ representing the eigenvalues and the vectors $\mathbf{a}$ and $\mathbf{b}$ representing the eigenvectors. Rewriting Eqs. (55) and (56) as a 6 by 6 matrix formed from the four 3 by 3 matrices that appear in (55) and (56) and also representing the two $3 \mathrm{D}$ vectors $\mathbf{a}$ and $\mathbf{b}$ as one $6 \mathrm{D}$ vector, the following representation is obtained:

$$
\left[\begin{array}{cc}
\mathbf{Q}-\rho v^{2} \mathbf{1} & \mathbf{C}-\rho_{f} v^{2} \mathbf{1} \\
\mathbf{C}^{T}-\rho_{f} v^{2} \mathbf{1} & M \mathbf{n} \otimes \mathbf{n}-\rho_{f} v^{2}\left\{\mathbf{J}+\frac{i \mu}{\omega} \mathbf{R}\right\}
\end{array}\right]\left[\begin{array}{l}
\mathbf{a} \\
\mathbf{b}
\end{array}\right]=0 .
$$

The equation above (57) is a generalization of the Christoffel equation (Mason 1958; Auld 1973) in anisotropic elastic wave propagation to the poroelastic case, the poroelastic Christoffel equation is a possible name for this result. Since the right-hand side of this linear system of equations is a zero $6 \mathrm{D}$ vector, it follows from Cramer's rule that, in order to avoid the trivial solution, it is necessary to set the determinant of the 6 by 6 matrix equal to zero, thus

$$
\left|\begin{array}{cc}
\mathbf{Q}-\rho v^{2} \mathbf{1} & \mathbf{C}-\rho_{f} v^{2} \mathbf{1} \\
\mathbf{C}^{T}-\rho_{f} v^{2} \mathbf{1} & M \mathbf{n} \otimes \mathbf{n}-\rho_{f} v^{2}\left\{\mathbf{J}+\frac{i \mu}{\omega} \mathbf{R}\right\}
\end{array}\right|=0 .
$$

\subsection{Fabric dependence of tensors Q, C, J and R}

Formulas relating the acoustic tensor $\mathbf{Q}$, the flow resistivity tensor $\mathbf{R}$ and the tensor $\mathbf{C}$, representing the interaction of the velocity fields $\mathbf{u}$ and $\mathbf{w}$, to the fabric tensor $\mathbf{F}$ are obtained in this subsection.

The dependence of the elastic acoustic tensor $\mathbf{Q}$ upon the fabric tensor $\mathbf{F}$ is obtained by substituting (49) into the first of (54); the result is presented in the following paragraph in three different, but equivalent, notations.

$$
\begin{aligned}
Q_{i k}= & \left(c_{1}^{c d}+c_{2}^{c d} F_{m j} n_{m} n_{j}+c_{3}^{c d} F_{m r} F_{r j} n_{m} n_{j}\right) \delta_{k i} \\
& +q_{1} n_{i} n_{k}+c_{2}^{c d} F_{k i}+q_{2}\left(F_{i j} n_{j} n_{k}+F_{k m} n_{m} n_{i}\right) \\
& +c_{3}^{c d}\left(F_{i r} F_{r k}+F_{k r} F_{r j} n_{j} n_{i}+F_{i r} F_{r m} n_{m} n_{k}\right) \\
& +q_{3}\left(F_{k q} F_{q m} n_{m} n_{i}+F_{i q} F_{q j} n_{j} n_{k}\right) \\
& +q_{4} F_{i j} n_{j} F_{k m} n_{m}+q_{5}\left(F_{i j} n_{j} F_{k q} F_{q m} n_{m}\right. \\
& \left.+F_{k m} n_{m} F_{i q} F_{q j} n_{j}\right)+q_{6} F_{i s} F_{s j} n_{j} F_{k q} F_{q m} n_{m} \\
\mathbf{Q}= & \left(c_{1}^{c d}+c_{2}^{c d} t r\{\mathbf{F} \cdot \mathbf{n} \otimes \mathbf{n}\}+c_{3}^{c d} t r\left\{\mathbf{F}^{2} \cdot \mathbf{n} \otimes \mathbf{n}\right\}\right) \mathbf{1} \\
& +q_{1} \mathbf{n} \otimes \mathbf{n}+c_{2}^{c d} \mathbf{F}+q_{2}(\mathbf{F} \cdot \mathbf{n} \otimes \mathbf{n}+\mathbf{n} \otimes \mathbf{n} \cdot \mathbf{F}) \\
& +c_{3}^{c d} \mathbf{F}^{2}+q_{3}\left(\mathbf{F}^{2} \cdot \mathbf{n} \otimes \mathbf{n}+\mathbf{n} \otimes \mathbf{n} \cdot \mathbf{F}^{2}\right) \\
& +q_{4} \mathbf{F} \cdot \mathbf{n} \otimes \mathbf{F} \cdot \mathbf{n} \\
& +q_{5}\left(\mathbf{F} \cdot \mathbf{n} \otimes \mathbf{F}^{2} \cdot \mathbf{n}+\mathbf{F}^{2} \cdot \mathbf{n} \otimes \mathbf{F} \cdot \mathbf{n}\right) \\
& +q_{6} \mathbf{F}^{2} \cdot \mathbf{n} \otimes \mathbf{F}^{2} \cdot \mathbf{n}
\end{aligned}
$$


and in the principal coordinate system of the fabric tensor,

$$
\begin{aligned}
& \mathbf{Q}=\left(c_{1}^{c d}+c_{2}^{c d}\left(F_{1} n_{1}^{2}+F_{2} n_{2}^{2}+F_{3} n_{3}^{2}\right)\right. \\
& \left.+c_{3}^{c d}\left(F_{1}^{2} n_{1}^{2}+F_{2}^{2} n_{2}^{2}+F_{3}^{2} n_{3}^{2}\right)\right)\left[\begin{array}{lll}
1 & 0 & 0 \\
0 & 1 & 0 \\
0 & 0 & 1
\end{array}\right] \\
& +q_{1}\left[\begin{array}{ccc}
n_{1}^{2} & n_{1} n_{2} & n_{1} n_{3} \\
n_{1} n_{2} & n_{2}^{2} & n_{2} n_{3} \\
n_{1} n_{3} & n_{2} n_{3} & n_{3}^{2}
\end{array}\right]+c_{2}^{c d}\left[\begin{array}{ccc}
F_{1} & 0 & 0 \\
0 & F_{2} & 0 \\
0 & 0 & F_{3}
\end{array}\right] \\
& +q_{2}\left[\begin{array}{ccc}
2 F_{1} n_{1}^{2} & \left(F_{1}+F_{2}\right) n_{1} n_{2} & \left(F_{1}+F_{3}\right) n_{1} n_{3} \\
\left(F_{1}+F_{2}\right) n_{1} n_{2} & 2 F_{2} n_{2}^{2} & \left(F_{2}+F_{3}\right) n_{2} n_{3} \\
\left(F_{1}+F_{3}\right) n_{1} n_{3} & \left(F_{2}+F_{3}\right) n_{2} n_{3} & 2 F_{3} n_{3}^{2}
\end{array}\right] \\
& +c_{3}^{c d}\left[\begin{array}{ccc}
F_{1}^{2} & 0 & 0 \\
0 & F_{2}^{2} & 0 \\
0 & 0 & F_{3}^{2}
\end{array}\right] \\
& +q_{3}\left[\begin{array}{ccc}
2 F_{1}^{2} n_{1}^{2} & \left(F_{1}^{2}+F_{2}^{2}\right) n_{1} n_{2} & \left(F_{1}^{2}+F_{3}^{2}\right) n_{1} n_{3} \\
\left(F_{1}^{2}+F_{2}^{2}\right) n_{1} n_{2} & 2 F_{2}^{2} n_{2}^{2} & \left(F_{2}^{2}+F_{3}^{2}\right) n_{2} n_{3} \\
\left(F_{1}^{2}+F_{3}^{2}\right) n_{1} n_{3} & \left(F_{2}^{2}+F_{3}^{2}\right) n_{2} n_{3} & 2 F_{3}^{2} n_{3}^{2}
\end{array}\right] \\
& +q_{4}\left[\begin{array}{ccc}
F_{1}^{2} n_{1}^{2} & F_{1} F_{2} n_{1} n_{2} & F_{1} F_{3} n_{1} n_{3} \\
F_{1} F_{2} n_{1} n_{2} & F_{2}^{2} n_{2}^{2} & F_{2} F_{3} n_{2} n_{3} \\
F_{1} F_{3} n_{1} n_{3} & F_{2} F_{3} n_{2} n_{3} & F_{3}^{2} n_{3}^{2}
\end{array}\right] \\
& +q_{5}\left[\begin{array}{ccc}
2 F_{1}^{3} n_{1}^{2} & \left(F_{1} F_{2}^{2}+F_{2} F_{1}^{2}\right) n_{1} n_{2} & \left(F_{1} F_{3}^{2}+F_{3} F_{1}^{2}\right) n_{1} n_{3} \\
\left(F_{1} F_{2}^{2}+F_{2} F_{1}^{2}\right) n_{1} n_{2} & 2 F_{2}^{3} n_{2}^{2} & \left(F_{3} F_{2}^{2}+F_{2} F_{3}^{2}\right) n_{2} n_{3} \\
\left(F_{1} F_{3}^{2}+F_{3} F_{1}^{2}\right) n_{1} n_{3} & \left(F_{3} F_{2}^{2}+F_{2} F_{3}^{2}\right) n_{2} n_{3} & 2 F_{3}^{3} n_{3}^{2}
\end{array}\right] \\
& +q_{6}\left[\begin{array}{ccc}
F_{1}^{4} n_{1}^{2} & F_{1}^{2} F_{2}^{2} n_{1} n_{2} & F_{1}^{2} F_{3}^{2} n_{1} n_{3} \\
F_{1}^{2} F_{2}^{2} n_{1} n_{2} & F_{2}^{4} n_{2}^{2} & F_{2}^{2} F_{3}^{2} n_{2} n_{3} \\
F_{1}^{2} F_{3}^{2} n_{1} n_{3} & F_{2}^{2} F_{3}^{2} n_{2} n_{3} & F_{3}^{4} n_{3}^{2}
\end{array}\right],
\end{aligned}
$$

where

$$
\begin{aligned}
& q_{1}=c_{1}^{c d}+a_{1}^{c d}+M \frac{\left(3 K^{m}-a_{o}^{d}\right)^{2}}{\left(3 K^{m}\right)^{2}}, \\
& q_{2}=c_{2}^{c d}+a_{2}^{c d}-\frac{M\left(3 K^{m}-a_{o}^{d}\right) a_{I}^{d}}{\left(3 K^{m}\right)^{2}}, \\
& q_{3}=c_{3}^{c d}+a_{3}^{c d}-M \frac{\left(3 K^{m}-a_{o}^{d}\right) a_{I I}^{d}}{\left(3 K^{m}\right)^{2}} \\
& q_{4}=b_{1}^{c d}+M \frac{\left(a_{I}^{d}\right)^{2}}{\left(3 K^{m}\right)^{2}}, \\
& q_{5}=b_{2}^{c d}+M \frac{a_{I}^{d} a_{I I}^{d}}{\left(3 K^{m}\right)^{2}}
\end{aligned}
$$

$q_{6}=b_{3}^{c d}+M \frac{\left(a_{I I}^{d}\right)^{2}}{\left(3 K^{m}\right)^{2}}$.

The six quantities defined in (60) are scalar-valued functions of $\phi, I I$ and $I I I$.

The formula for the tensor $\mathbf{C}$ is obtained by substituting (25) into (54) and then employing (44), thus

$$
C_{i k}=M n_{i} n_{k}-\frac{M}{3 K^{m}}\left\{a_{o}^{c d} n_{i}+a_{I}^{c d} F_{i j} n_{j}+a_{I I}^{c d} F_{i q} F_{q j} n_{j}\right\} n_{k} .
$$

The micro-macro velocity average tensor $\mathbf{J}$ is related to the fabric by

$$
J_{i j}=j_{1} \delta_{i j}+j_{2} F_{i j}+j_{3} F_{i q} F_{q j}
$$

where $j_{1}, j_{2}$, and $j_{3}$ are functions of $\phi, I I$ and $I I I$. Similarly, The flow-resistivity tensor $\mathbf{R}$, is related to the fabric by

$R_{i j}=r_{1} \delta_{i j}+r_{2} F_{i j}+r_{3} F_{i q} F_{q j}$,

where $r_{1}, r_{2}$, and $r_{3}$ are functions of $\phi, I I$ and $I I I$, and $\mathbf{R}$ is equivalent to the inverse of the second-rank intrinsic permeability tensor $\mathbf{K}$.

\subsection{Tortuosity, fabric, frequency dependence}

The relationship between the second-rank intrinsic permeability tensor K, introduced in Darcy's Law, Eq. (11), and the fabric tensor $\mathbf{F}$ is obtained by assuming that $\mathbf{K}$ is an isotropic function of $\mathbf{F}$. The relationship between two second-rank symmetric tensors in which one is an isotropic function of the other then produces the relationship

$K_{i j}=k_{1} \delta_{i j}+k_{2} F_{i j}+k_{3} F_{i q} F_{q j}$.

This permeability tensor can be rewritten using the intrinsic permeability $\kappa_{0}$ as

$K_{i j}=\kappa_{0}\left(K_{1} \delta_{i j}+K_{2} F_{i j}+K_{3} F_{i q} F_{q j}\right)$.

where $K_{i j}$ is the intrinsic permeability which is representative of the geometry of the porous medium only, not the fluid, and $K_{1}, K_{2}$, and $K_{3}$ are functions of $\phi, I I$ and $I I I$. The hydraulic permeability $K_{i j} / \mu$ differs from the intrinsic permeability where $\mu$ is the pore fluid viscosity. We introduce the symbol $\kappa_{0}$ to represent the value of the intrinsic permeability tensor when it is averaged over all possible directions at a point,

$$
\kappa_{0}=\frac{\pi^{2}}{2}\left(2 K_{1}+K_{2} \operatorname{tr} \mathbf{F}+K_{3} \operatorname{tr}\left[\mathbf{F} \cdot \mathbf{F}^{T}\right]\right)
$$


and rewrite (64) as

$K_{i j}=\kappa_{0}\left(K_{1}^{A} \delta_{i j}+K_{2}^{A} F_{i j}+K_{3}^{A} F_{i q} F_{q j}\right)$,

where

$K_{i}^{A}=\frac{K_{i}}{\kappa_{0}}, \quad i=1,2,3$.

The fabric tensor describes the configuration and orientation of the flow paths and the average intrinsic permeability $\kappa_{0}$ is proportional to the squared average diameter of the pores, $d$, through which the fluid moves:

$\kappa_{0} \propto d^{2}$.

The tensor $\mathbf{K}$ takes into account dissipation phenomena due to viscous losses as shown in (53); however, this expression (65a) for permeability is adequate only for low frequencies of fluid motion and needs to be corrected to take into account the change in fluid flow regime occurring between low and high frequencies of wave propagation. This correction was originally introduced by Johnson et al. (1987) describing a dynamic permeability in a porous medium system characterized by orthogonally intersected tubes.

$$
\begin{aligned}
K_{i j}(\omega)=\kappa_{0} & {\left[1-2 \frac{\mathrm{J}_{1}(d \chi)}{d \chi \mathrm{J}_{0}(d \chi)}\right] } \\
& \times\left(K_{1} \delta_{i j}+K_{2} F_{i j}+K_{3} F_{i q} F_{q j}\right) .
\end{aligned}
$$

The dynamic permeability tensor $\mathbf{K}$ is then described as a function of the average intrinsic permeability $\kappa_{0}$, the fabric tensor and Bessel functions that characterize the dynamics of the oscillatory fluid flow inside a cylindrical channel. In this equation, $J_{1}$ and $J_{0}$ are, respectively, the first order and zeroth order Bessel functions of the first kind; $d$ corresponds to the average characteristic pore dimension; and the inverse of the viscous skin depth $\chi$ is defined as a function of the angular frequency $\omega$, the fluid mass density $\rho^{f}$ and the dynamic viscosity of the fluid $\mu$ :

$\chi=\left(\frac{i \omega \rho^{f}}{\mu}\right)^{1 / 2}$.

The motion of the viscous fluid relative to the solid is characterized by the velocity gradient profile perpendicular to the pore wall, as a consequence of the viscous properties of the fluid. If the characteristic thickness of the viscous layer (viscous skin depth) is greater than the pore diameter $d$, the resulting velocity profile will be parabolic throughout the pore lumen and the fluid flow will be Poiseuille flow. However, as the frequency increases, the viscous skin depth becomes smaller than the pore radius, and the profile of the fluid flow is no longer parabolic. A consequence of this change in the fluid flow regime into the pore is the existence of a critical frequency $f_{\text {crit }}=\mu / \pi \rho^{f} d^{2}$, distinguishing the frequency regions where the slow wave may theoretically propagate. In the low-frequency regime (below $\omega_{\text {crit }}=2 \pi f_{\text {crit }}$ ), the viscous coupling phenomenon dominates over the inertial one, locking together fluid and solid displacements, and thus hampering the genesis of the slow wave. However, at high frequencies, the viscous coupling phenomenon becomes much less important than the inertial one, and the relative movement between fluid and solid is no longer impeded. Above this critical frequency, both fast and slow waves may be expected to propagate.

The inverse of the second-rank intrinsic permeability tensor $\mathbf{K}$, the flow-resistivity tensor $\mathbf{R}$, is related to the fabric in the situation representing the frequency dependence by (63) where

$$
\begin{aligned}
& r_{i}(\omega, \phi, I I, I I I)=\frac{r_{i}^{A}(\phi, I I, I I I)}{\kappa_{0}\left[1-2 \frac{\mathrm{J}_{1}(d \chi)}{d \chi \mathrm{J}_{0}(d \chi)}\right]}, \\
& R_{i j}(\omega)=\frac{1}{\kappa_{0}\left[1-2 \frac{\mathrm{J}_{1}(d \chi)}{d \chi \mathrm{J}_{0}(d \chi)}\right]}\left(r_{1} \delta_{i j}+r_{2} F_{i j}+r_{3} F_{i q} F_{q j}\right),
\end{aligned}
$$

where the $r_{i}^{A}, i=1,2,3$, are functions of $\phi, I I$ and III. Following Johnson et al. (1987) and Perrot et al. (2008) the tortuosity tensor $\mathbf{A}(\omega)$ is introduced

$\rho_{f} \mathbf{A}(\omega) \cdot \frac{\partial \mathbf{v}}{\partial t}=-\nabla p, \quad\left(\rho_{f} A_{i j}(\omega) \frac{\partial v_{j}}{\partial t}=-\frac{\partial p}{\partial x_{i}}\right)$,

and using Darcy's law (26) it follows that,

$\mathbf{A}(\omega)=\frac{i \mu \phi}{\omega \rho_{o}} \mathbf{K}^{-1}(\omega)=\frac{i \mu \phi}{\omega \rho_{o}} \mathbf{R}(\omega)$,

if we assume that $\mathbf{v}=$ constant $\cdot e^{-i \omega t}$. From (62) and (70) it follows that

$A_{i j}(\omega)=\frac{i \mu \phi}{\omega \rho_{o}}\left(r_{1} \delta_{i j}+r_{2} F_{i j}+r_{3} F_{i q} F_{q j}\right)$,

establishing a connection between the tortuosity tensor and the fabric tensor in the case of harmonic wave propagation, where the $r_{i}$ are given by (69). We have not explored this relationship yet, but we anticipate that this relationship will yield relationships between the tortuosity tensor and the curvature and torsion of the fluid channels in the porous material.

\section{Propagation of waves along the principal axes of symmetry in orthotropic porous media}

\subsection{Phase velocity and phase direction}

In this section, the solution is developed for waves that propagate in the direction of a principal axis of the fabric tensor. 
The direction of propagation is selected to be the one direction, thus $\mathbf{F}$ and $\mathbf{n}$ are given by

$\mathbf{n}=\{1,0,0\} \quad$ and $\quad \mathbf{F}=\left[\begin{array}{ccc}F_{1} & 0 & 0 \\ 0 & F_{2} & 0 \\ 0 & 0 & F_{3}\end{array}\right]$,

and the displacement vectors reduce to

$\mathbf{u}(\mathbf{x}, t)=\mathbf{a} e^{i \omega\left(\frac{x_{1}}{v}-t\right)}=\mathbf{a} e^{i\left((k+i \alpha) x_{1}-\omega t\right)}=\mathbf{a} e^{-\alpha x_{1}} e^{i\left(k x_{1}-\omega t\right)}$,

$w(\mathbf{x}, t)=\mathbf{b} e^{i \omega\left(\frac{x_{1}}{v}-t\right)}=\mathbf{b} e^{i\left((k+i \alpha) x_{1}-\omega t\right)}=\mathbf{b} e^{-\alpha x_{1}} e^{i\left(k x_{1}-\omega t\right)}$.

The solution to the problem is the solution of the 6 by 6 system of equations given by (58), thus the values of the tensors $\mathbf{J}, \mathbf{R}, \mathbf{C}$ and $\mathbf{Q}$ in the coordinate system of the principal axes of the fabric tensor and at the vector $\mathbf{n}=\{1,0,0\}$ are determined first. Under these conditions, $\mathbf{J}$ and $\mathbf{R}$ are given by

$\mathbf{J}=\left[\begin{array}{ccc}J_{11} & 0 & 0 \\ 0 & J_{22} & 0 \\ 0 & 0 & J_{33}\end{array}\right], \quad \mathbf{R}=\left[\begin{array}{ccc}R_{11} & 0 & 0 \\ 0 & R_{22} & 0 \\ 0 & 0 & R_{33}\end{array}\right]$,

where $\mathbf{R}$ and $\mathbf{J}$ are given by (62) and (63), thus

$\begin{aligned} \mathbf{J} & =\left[\begin{array}{ccc}j_{1}+j_{2} F_{1}+j_{3} F_{1}^{2} & 0 & 0 \\ 0 & j_{1}+j_{2} F_{2}+j_{3} F_{2}^{2} & 0 \\ 0 & 0 & j_{1}+j_{2} F_{3}+j_{3} F_{3}^{2}\end{array}\right], \\ \mathbf{R} & =\left[\begin{array}{ccc}r_{1}+r_{2} F_{1}+r_{3} F_{1}^{2} & 0 & 0 \\ 0 & r_{1}+r_{2} F_{2}+r_{3} F_{2}^{2} & 0 \\ 0 & 0 & r_{1}+r_{2} F_{3}+r_{3} F_{3}^{2}\end{array}\right]\end{aligned}$

C is given by (61) as

$C_{i k}=M n_{i} n_{k}-\frac{M}{3 K^{m}}\left\{a_{o}^{c d} n_{i}+a_{I}^{c d} F_{i j} n_{j}+a_{I I}^{c d} F_{i q} F_{q j} n_{j}\right\} n_{k}$,

$$
\mathbf{C}=\left[\begin{array}{ccc}
C_{11} & 0 & 0 \\
0 & 0 & 0 \\
0 & 0 & 0
\end{array}\right]
$$

and $\mathbf{Q}$ is given by (59c) as

$$
\begin{aligned}
\mathbf{Q}= & \left(c_{1}^{c d}+c_{2}^{c d} F_{1}+c_{3}^{c d} F_{1}^{2}\right)\left[\begin{array}{ccc}
1 & 0 & 0 \\
0 & 1 & 0 \\
0 & 0 & 1
\end{array}\right]+q_{1}\left[\begin{array}{lll}
1 & 0 & 0 \\
0 & 0 & 0 \\
0 & 0 & 0
\end{array}\right] \\
& +c_{2}^{c d}\left[\begin{array}{ccc}
F_{1} & 0 & 0 \\
0 & F_{2} & 0 \\
0 & 0 & F_{3}
\end{array}\right]+q_{2}\left[\begin{array}{ccc}
2 F_{1} & 0 & 0 \\
0 & 0 & 0 \\
0 & 0 & 0
\end{array}\right]
\end{aligned}
$$

$$
\begin{aligned}
& +c_{3}^{c d}\left[\begin{array}{ccc}
F_{1}^{2} & 0 & 0 \\
0 & F_{2}^{2} & 0 \\
0 & 0 & F_{3}^{2}
\end{array}\right]+q_{3}\left[\begin{array}{ccc}
2 F_{1}^{2} & 0 & 0 \\
0 & 0 & 0 \\
0 & 0 & 0
\end{array}\right] \\
& +q_{4}\left[\begin{array}{ccc}
F_{1}^{2} & 0 & 0 \\
0 & 0 & 0 \\
0 & 0 & 0
\end{array}\right]+2 q_{5}\left[\begin{array}{ccc}
I F_{1}^{2}-I I F_{1}+I I I & 0 & 0 \\
0 & 0 & 0 \\
0 & 0 & 0
\end{array}\right] \\
& +q_{6}\left[\begin{array}{cccc}
(I \bullet I-I I) F_{1}^{2}-(I \bullet I I-I I I) F_{1}+I \bullet I I I & 0 & 0 \\
0 & 0 & 0 \\
0 & 0 & 0
\end{array}\right] \\
& =\left[\begin{array}{ccc}
Q_{11} & 0 & 0 \\
0 & Q_{22} & 0 \\
0 & 0 & Q_{33}
\end{array}\right],
\end{aligned}
$$

where

$Q_{11}=q_{o}^{11}+q_{1}^{11} F_{1}+q_{2}^{11} F_{1}^{2}$,

$Q_{22}=c_{1}^{c d}+c_{2}^{c d}\left(F_{2}+F_{1}\right)+c_{3}^{c d}\left(F_{2}^{2}+F_{1}^{2}\right)$

$Q_{33}=c_{1}^{c d}+c_{2}^{c d}\left(F_{3}+F_{1}\right)+c_{3}^{c d}\left(F_{3}^{2}+F_{1}^{2}\right)$,

and where

$q_{o}^{11}=c_{1}^{c d}+q_{1}+2 I I I q_{5}+q_{6} I \bullet I I I$,

$q_{1}^{11}=2 c_{2}^{c d}+2 q_{2}-2 I I q_{5}-q_{6}(I \bullet I I-I I I)$,

$q_{2}^{11}=2 c_{3}^{c d}+2 q_{3}+q_{4}+2 I q_{5}+(I \bullet I-I I) q_{6}$.

In the coordinate system of the principal axes of the fabric tensor and at the vector $\mathbf{n}=\{1,0,0\}$, the four 3 by 3 submatrices that form the 6 by 6 matrix in Eq. (58) are given by

$$
\begin{aligned}
\mathbf{Q}-\rho v^{2} \mathbf{1} & =\left[\begin{array}{ccc}
Q_{11}-\rho v^{2} & 0 & 0 \\
0 & Q_{22}-\rho v^{2} & 0 \\
0 & 0 & Q_{33}-\rho v^{2}
\end{array}\right] \\
\mathbf{C}-\rho_{f} v^{2} \mathbf{1} & =\mathbf{C}^{T}-\rho_{f} v^{2} \mathbf{1} \\
& =\left[\begin{array}{ccc}
C_{11}-\rho_{f} v^{2} & 0 & 0 \\
0 & -\rho_{f} v^{2} & 0 \\
0 & 0 & -\rho_{f} v^{2}
\end{array}\right]
\end{aligned}
$$




$$
\begin{aligned}
& M \mathbf{n} \otimes \mathbf{n}-\rho_{f} v^{2}\left\{\mathbf{J}+\frac{i \mu}{\omega} \mathbf{R}\right\} \\
& =\left[\begin{array}{ccc}
M-\rho_{f} v^{2}\left\{J_{11}+\frac{i \mu}{\omega} R_{11}\right\} & 0 & 0 \\
0 & -\rho_{f} v^{2}\left\{J_{22}+\frac{i \mu}{\omega} R_{22}\right\} & 0 \\
0 & 0 & -\rho_{f} v^{2}\left\{J_{33}+\frac{i \mu}{\omega} R_{33}\right\}
\end{array}\right] .
\end{aligned}
$$

Substitution of the four 3 by 3 matrices above into the 6 by 6 determinant (58) reveals that the result may be expressed as three 2 by 2 matrices for the three sets of components, $\left\{a_{1}, b_{1}\right\},\left\{a_{2}, b_{2}\right\}$ and $\left\{a_{3}, b_{3}\right\}$;

$$
\begin{aligned}
& {\left[\begin{array}{cc}
Q_{11}-\rho v^{2} & C_{11}-\rho_{f} v^{2} \\
C_{11}-\rho_{f} v^{2} & M-\rho_{f} v^{2}\left\{J_{11}+\frac{i \mu}{\omega} R_{11}\right\}
\end{array}\right]\left[\begin{array}{l}
a_{1} \\
b_{1}
\end{array}\right]=0,} \\
& {\left[\begin{array}{cc}
Q_{22}-\rho v^{2} & -\rho_{f} v^{2} \\
-\rho_{f} v^{2} & -\rho_{f} v^{2}\left\{J_{22}+\frac{i \mu}{\omega} R_{22}\right\}
\end{array}\right]\left[\begin{array}{l}
a_{2} \\
b_{2}
\end{array}\right]=0,} \\
& {\left[\begin{array}{cc}
Q_{33}-\rho v^{2} & -\rho_{f} v^{2} \\
-\rho_{f} v^{2} & -\rho_{f} v^{2}\left\{J_{33}+\frac{i \mu}{\omega} R_{33}\right\}
\end{array}\right]\left[\begin{array}{l}
a_{3} \\
b_{3}
\end{array}\right]=0 .}
\end{aligned}
$$

Requiring that the determinants of these 2 by 2 matrices vanish yields four non-trivial solutions for the squared wave speed $v^{2}$. The vanishing of the first of the determinants of these 2 by 2 matrices provides two roots of a quadratic equation that represent the fast and the slow squared longitudinal wave speeds. These speeds are given by

$v^{2}=\frac{\omega^{2}}{\left(k_{1}+i \alpha_{1}\right)^{2}}$,

where the specific formulas for calculating $k_{1}$ and $\alpha_{1}$ require a hierarchy of substitutions specified by the following equations and the interposed text. $k_{1}$ and $\alpha_{1}$ are given by

$k_{1}=\frac{1}{\sqrt{2}} \sqrt{\sqrt{k_{o}^{2}+\alpha_{o}^{2}}+k_{o}}, \alpha_{1}=\frac{s g n \alpha_{o}}{\sqrt{2}} \sqrt{\sqrt{k_{o}^{2}+\alpha_{o}^{2}}-k_{o}}$,

where the two possible values of both $k_{o}$ and $\alpha_{o}$ are given by where the two possible values are determined by selecting the + and - signs. The two roots correspond to the fast and the slow longitudinal waves. The $k_{-1}$ and $\alpha_{-1}$ appearing in the formulas for $k_{o}$ and $\alpha_{o}$ above are given by

$$
\begin{aligned}
k_{-1} & =\frac{1}{\sqrt{2}} \sqrt{\sqrt{k_{-2}^{2}+\alpha_{-2}^{2}}+k_{-2}}, \\
\alpha_{-1} & =\frac{\operatorname{sgn} \alpha_{-2}}{\sqrt{2}} \sqrt{\sqrt{k_{-2}^{2}+\alpha_{-2}^{2}}+k_{-2}},
\end{aligned}
$$

where the $k_{-2}$ and $\alpha_{-2}$ appearing in the aforementioned equation are given by

$$
\begin{aligned}
k_{-2}= & \left(\frac{\rho}{\rho_{f}} M\right)^{2}+2 C_{11}^{2} \frac{\rho}{\rho_{f}} M \\
& +2 J_{11}\left(Q_{11}\left(\frac{\rho}{\rho_{f}} M-C_{11}^{2}\right)+2 C_{11}^{2} \frac{\rho}{\rho_{f}}\right) \\
& +\left(Q_{11}\right)^{2}\left\{\left(J_{11}\right)^{2}-\left(\frac{\mu}{\omega} R_{11}\right)^{2}\right\} \\
\alpha_{-2}= & 2 \frac{\mu}{\omega} R_{11}\left(Q_{11}\left(\frac{\rho}{\rho_{f}} M-C_{11}^{2}\right)+J_{11}+2 C_{11}^{2} \frac{\rho}{\rho_{f}}\right) .
\end{aligned}
$$

Fortunately, the formulas for the shear waves are much simpler. The vanishing of the second and third of the determinants of the 2 by 2 matrices in (86) provide a zero root and a non-zero root from each determinant. The two non-zero roots are the squared shear wave speeds

$$
\begin{aligned}
& v^{2}=\frac{Q_{22}}{\rho} \frac{J_{22}+\frac{i \mu}{\omega} R_{22}}{J_{22}-\frac{\rho_{f}}{\rho}+\frac{i \mu}{\omega} R_{22}} \text { and } \\
& v^{2}=\frac{Q_{33}}{\rho} \frac{J_{33}+\frac{i \mu}{\omega} R_{33}}{J_{33}-\frac{\rho_{f}}{\rho}+\frac{i \mu}{\omega} R_{33}} .
\end{aligned}
$$

$$
\begin{gathered}
k_{o}=\frac{2 \rho \omega^{2}\left[\left(J_{11}-\frac{\rho_{f}}{\rho}\right)\left(\frac{\rho}{\rho_{f}} M+J_{11}-2 C_{11} \pm k_{-1}\right)+\frac{\mu}{\omega} R_{11}\left(\frac{\mu}{\omega} R_{11} Q_{11} \pm \alpha_{-1}\right)\right]}{\left(\frac{\rho}{\rho_{f}} M+J_{11}-2 C_{11} \pm k_{-1}\right)^{2}+\left(\frac{\mu}{\omega} R_{11} Q_{11} \pm \alpha_{-1}\right)^{2}} \\
+\alpha_{o}=\frac{2 \rho \omega^{2}\left[\frac{\mu}{\omega} R_{11}\left(\frac{\rho}{\rho_{f}} M+J_{11}-2 C_{11} \pm k_{-1}\right)-\left(\frac{\mu}{\omega} R_{11} Q_{11} \pm \alpha_{-1}\right)\left(J_{11}-\frac{\rho_{f}}{\rho}\right)\right]}{\left(\frac{\rho}{\rho_{f}} M+J_{11}-2 C_{11} \pm k_{-1}\right)^{2}+\left(\frac{\mu}{\omega} R_{11} Q_{11} \pm \alpha_{-1}\right)^{2}},
\end{gathered}
$$


When the first of these are recast in the form of (51), $k_{2}$ and $\alpha_{2}$ are given by

$$
\begin{aligned}
k_{2} & =\frac{\omega}{\sqrt{2 \frac{Q_{22}}{\rho}}} \\
& \times \sqrt{\sqrt{\left\{\left(1-\frac{\frac{\rho_{f}}{\rho} J_{22}}{J_{22}^{2}+\left(\frac{\mu}{\omega} R_{22}\right)^{2}}\right)^{2}+\frac{\left(\frac{\rho_{f}}{\rho} \frac{\mu}{\omega} R_{22}\right)^{2}}{\left(J_{22}^{2}+\left(\frac{\mu}{\omega} R_{22}\right)^{2}\right)^{2}}+\left(1-\frac{\frac{\rho_{f}}{\rho} J_{22}}{J_{22}^{2}+\left(\frac{\mu}{\omega} R_{22}\right)^{2}}\right)\right.}} \\
\alpha_{2} & =\frac{\omega}{\sqrt{2 \frac{Q_{22}}{\rho}}} \\
& \times \sqrt{\sqrt{\left\{\left(1-\frac{\frac{\rho_{f}}{\rho} J_{22}}{J_{22}^{2}+\left(\frac{\mu}{\omega} R_{22}\right)^{2}}\right)^{2}+\frac{\left(\frac{\rho_{f}}{\rho} \frac{\mu}{\omega} R_{22}\right)^{2}}{\left(J_{22}^{2}+\left(\frac{\mu}{\omega} R_{22}\right)^{2}\right)^{2}}\right\}}-\left(1-\frac{\frac{\rho_{f}}{\rho} J_{22}}{J_{22}^{2}+\left(\frac{\mu}{\omega} R_{22}\right)^{2}}\right)},
\end{aligned}
$$

and similar formulas apply for the recasting of the second of (88); one need only replace the 2's by 3's in Eq. (92).

\subsection{Wave polarization}

The vectors $\mathbf{a}$ and $\mathbf{b}$ for the fast and slow waves are given by

$\mathbf{a}=\left\{a_{1}, 0,0\right\} \mathbf{b}=\left\{b_{1}, 0,0\right\}$

where $a_{1}$ and $b_{1}$ are related by the following two equivalent expressions for the fast wave

$a_{1}=\frac{\rho_{f}\left(v_{o}^{2}+c_{o}^{2}-C_{11}\right)}{\rho\left(v_{o}^{2}+c_{o}^{2}\right)-\rho_{f} Q_{11}}$

$b_{1}=\frac{\left(J_{11}+\frac{i \mu}{\omega} R_{11}\right)\left(v_{o}^{2}+c_{o}^{2}\right)-M}{v_{o}^{2}+c_{o}^{2}-C_{11}} b_{1}$,

and the next two equivalent expressions for the slow wave,

$$
\begin{aligned}
& a_{1}=\frac{\rho_{f}\left(v_{o}^{2}-c_{o}^{2}\right)-\rho_{f} C_{11}}{\rho\left(v_{o}^{2}+c_{o}^{2}\right)-\rho_{f} Q_{11}} \\
& b_{1}=\frac{\left(J_{11}+\frac{i \mu}{\omega} R_{11}\right)\left(c_{o}^{2}-v_{o}^{2}\right)+M}{c_{o}^{2}-v_{o}^{2}+C_{11}} b_{1},
\end{aligned}
$$

and for the two shear waves by

$\mathbf{a}=\left\{0, a_{2}, 0\right\} \mathbf{b}=\left\{0, b_{2}, 0\right\}, a_{2}=-\left(J_{22}+\frac{i \mu}{\omega} R_{22}\right) b_{2}$,

and

$\mathbf{a}=\left\{0,0, a_{3}\right\}, \mathbf{b}=\left\{0,0, b_{3}\right\}, a_{3}=-\left(J_{33}+\frac{i \mu}{\omega} R_{33}\right) b_{3}$,

respectively.

\subsection{Numerical example applied to cancellous bone}

The anisotropic poroelastic model of wave propagation is now applied to the case of cancellous bone, and these numer- ical results will be used to analyze fast and slow wave velocity measurements from bovine and human bone samples previously reported (Cardoso et al. 2003). In order to apply the model to cancellous bone, values of the fluid and solid constituents of bone were obtained from the literature. Since the fluid saturating the cancellous bone structure in our experiments is water, the fluid mass density $\rho^{f}=1,000 \mathrm{Kg} / \mathrm{m}^{3}$, bulk modulus $K^{f}=2.25 \mathrm{GPa}$ and viscosity $\mu=1 \times 10^{-3}$ Pas. Furthermore, the mass density of the solid tissue in trabecular bone $\rho^{s}$, has been reported to vary between 1,800 and $2,200 \mathrm{Kg} / \mathrm{m}^{3}$ (Ashman and Rho 1988; Nicholson et al. 1997; Morgan et al. 2003) depending on the tissue mineral density of the sample being measured. Likewise, the Young's elastic modulus of the mineralized matrix, $E^{s}$, has been determined using acoustic microscopy and nanoindentation (Turner et al. 1999; Jorgensen and Kundu 2002; Rho et al. 1997, 1999; Roy et al. 1999; Zysset et al. 1999; Hoffler et al. 2000a,b; Hengsberger et al. 2001, 2002), exhibiting values ranging from 11.4 to $22.7 \mathrm{GPa}$. Specifically, the $E^{s}$ value measured in the circumferential direction varies between 13.5 and $16 \mathrm{GPa}$, and in the longitudinal direction the $E^{s}$ value varies between 19 and $23 \mathrm{GPa}$. These values of $E^{s}$ obtained at the subtrabecular scale are close to the classical values measured for cortical bone, which are $15 \mathrm{GPa}$ in the circumferential direction and $20 \mathrm{GPa}$ in the longitudinal direction. This large variability reported in the literature for $E^{s}$ is certainly due to the intrinsic variability of the tissue mineralization and organic composition, but may also be a consequence of using estimation approaches based on different assumptions (boundary conditions, geometry, homogeneity), and experimental conditions (temperature, tissue dehydration, strain rate, size scale). The values for the material properties of the solid and fluid constituents of bone considered in our numerical results are summarized in Table 1.

In addition to the material properties of the solid and fluid constituents of bone, the model requires the value of the average, or effective, pore diameter $d$ and the average intrinsic permeability $\kappa_{0}$ as a function of the porosity. Histomorphometrical studies on cancellous bone have reported pore

Table 1 Bone tissue modulus, mass density and fluid viscosity of fluid in bone

\begin{tabular}{llll}
\hline Parameter & Symbol & Value & Units \\
\hline Young's elastic modulus of the solid & $E^{\mathrm{s}}$ & 18 & $\mathrm{GPa}$ \\
Mass density of the solid & $\rho^{\mathrm{s}}$ & 2000 & $\mathrm{Kg} / \mathrm{m}^{3}$ \\
Mass density of the fluid & $\rho^{\mathrm{f}}$ & 1000 & $\mathrm{Kg} / \mathrm{m}^{3}$ \\
Fluid bulk modulus & $K^{\mathrm{f}}$ & 2.25 & $\mathrm{GPa}$ \\
$\begin{array}{l}\text { Fluid viscosity } \\
\begin{array}{l}\text { Pore size-porosity } \\
\text { proportionality constant in Eq. (96) }\end{array}\end{array}$ & $\mathrm{c}$ & $1 \times 10^{-3}$ & $\mathrm{Pas}$ \\
\hline
\end{tabular}



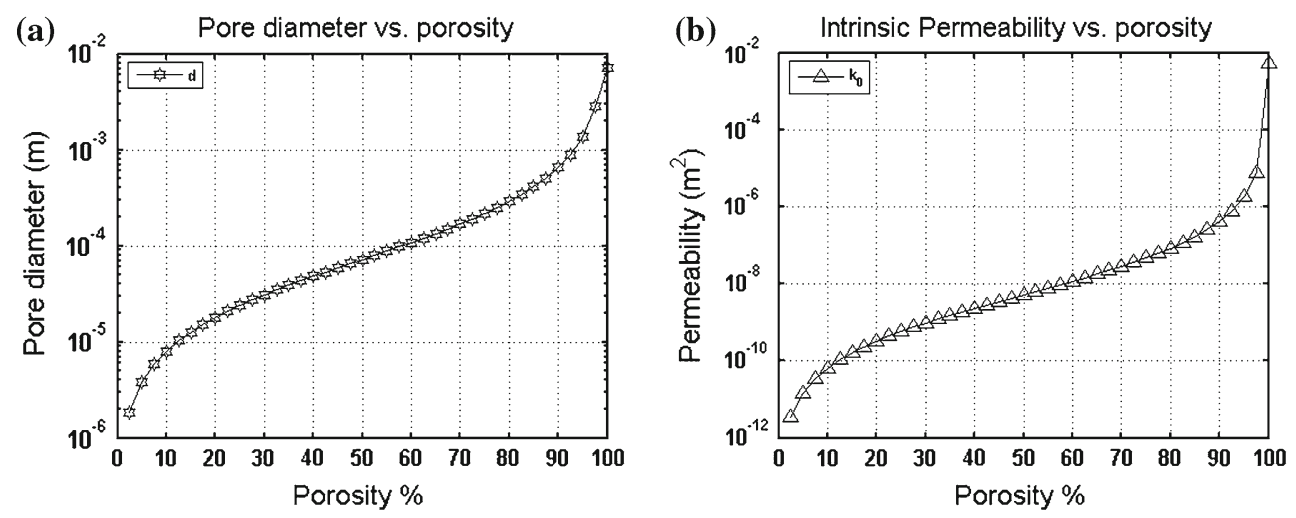

Fig. 1 Pore diameter $d$ as a function of porosity (a) and intrinsic permeability $\kappa_{0}$ as a function of porosity (b)

(a) Theoretical velocity of longitudinal waves - isotropy

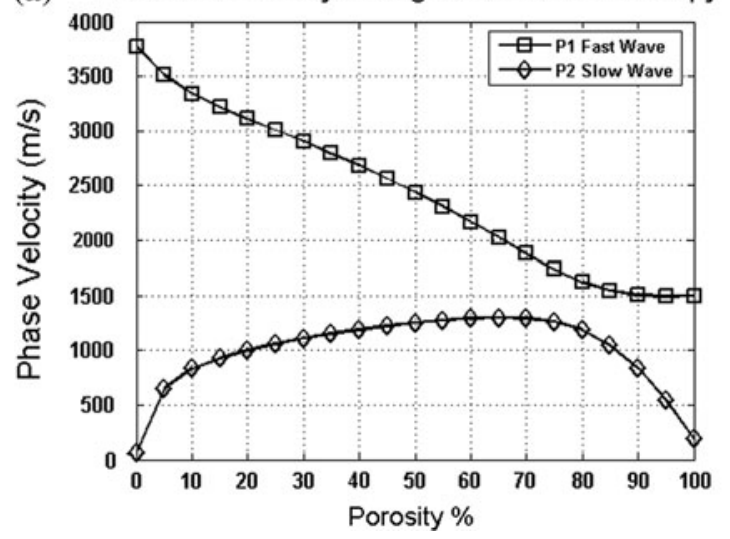

(b) Phase Velocity along the axes of symmetry - anisotropy

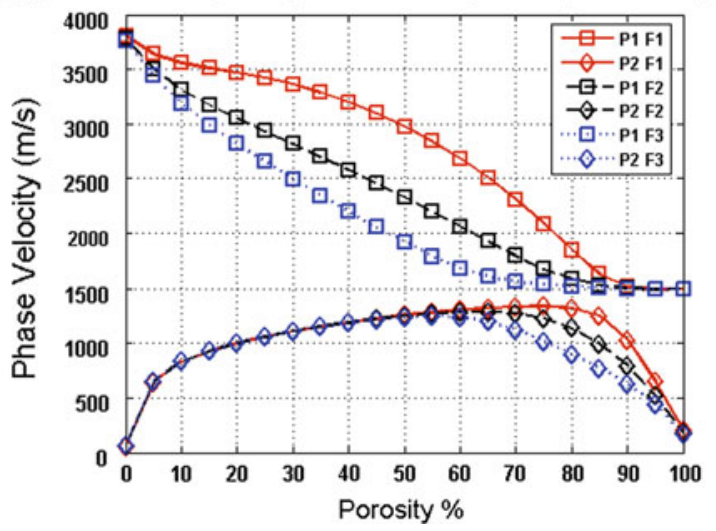

Fig. 2 Phase velocity as a function of porosity of the four wave modes in isotropic bone medium (a), and along the axes of symmetry in orthotropic bone sample (b)

sizes (trabecular spacing) ranging from 300 to 2, $200 \mu \mathrm{m}$ for samples between 52 and 96\% porosity (Parfitt et al. 1983; Rehman et al. 1994; Hildebrand et al. 1999; Glorieux et al. 2000). Furthermore, the pore size in $5-10 \%$ porosity cortical bone tissue is considered to vary around 20 to $60 \mu \mathrm{m}$, which corresponds to the pore size of Haversian canals (Jones et al. 2004; Basillais et al. 2007). Based on such bounds, the following empirical relationship for the pore diameter $d$ as a function of the porosity is proposed:

$d(\phi)=c[\phi /(1-\phi)]$

where $c$ is a constant of proportionality, in this study chosen equal to $5 \times 10^{-5} \mathrm{~m}$, which leads to a variation of the pore diameter as a function of porosity (Fig. 1a) that corresponds to the bounds previously mentioned. It is important to notice that this is just an approximation to relate the variation of the average pore size with the porosity, but that such relationship may be different and much more complex. This aspect would be further explored in the near future by the authors.

Based on the pore size-porosity relationship (97), the dependence of the intrinsic permeability on the effective pore size $\kappa_{0}=d^{2}$ (Bear 1988), can be transformed to be depen- dent on the porosity as well $\kappa_{0}(\phi)=c^{2}[\phi /(1-\phi)]^{2}$. Predictions of the intrinsic permeability are shown in Fig. 1b, and exhibit a large variability that coincides with studies reporting experimental measurements of the permeability $\kappa_{0}\left(1 \times 10^{-12}-1 \times 10^{-7} \mathrm{~m}^{2}\right)$ on cancellous bone (Lim and Hong 2000; Grimm and Williams 1997b; Nauman et al. 1999; Kohles et al. 2001; Kohles and Roberts 2002; Baroud et al. 2004; Beaudoin et al. 1991; Li et al. 1987). The variability of the intrinsic permeability in porous media is due to the dependence of the permeability on the porosity (Grimm and Williams 1997b; Nauman et al. 1999) and the microstructure of the sample (Nauman et al. 1999; Kohles et al. 2001; Kohles and Roberts 2002; Baroud et al. 2004).

\subsection{Phase velocity as a function of porosity}

The phase velocities of the two longitudinal modes of wave propagation along the axes of symmetry of an isotropic bone specimen (Fig. 2a) and an orthotropic bone specimen (Fig. 2b) are shown as functions of the porosity in Fig. 2. The fast wave velocity (squares) depicted in Fig. 2a linearly decrease as the porosity increases from zero to $80 \%$; 
(a) Theoretical and experimetal P1 \& P2 waves - isotropy

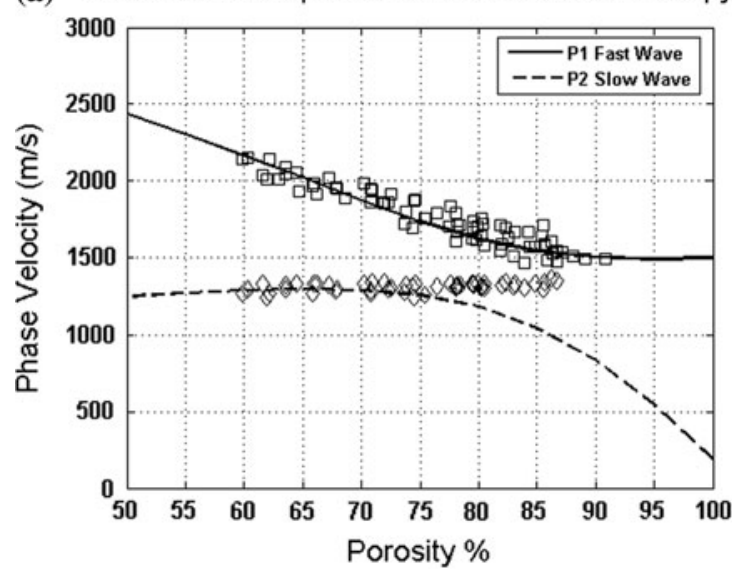

Fig. 3 a Fast (squares) and slow (diamonds) wave velocities averaged over three orthogonal directions on each sample plotted as a function of bone porosity, compared to the theoretical fast (solid) and slow wave velocities (dashed) in isotropic medium; b Fast wave velocity (squares) and slow wave velocity data (diamonds) measured

conversely, the slow wave velocity (diamonds) increase with the porosity within the same range of porosity. However, this monotonic behavior changes drastically for porosities higher than about $80 \%$, and the fast wave velocity becomes almost constant and independent of the porosity. At the same high porosity level, the slow wave velocity shows a clear inflexion, becoming inversely related to the porosity. It is important to notice that in addition to being independent of the porosity, the fast wave at high porosity exhibits velocity values equal to the wave propagation in the fluid saturating the pores, the velocity of sound in water $\left(v_{\text {fluid }}=1480-1500 \mathrm{~m} / \mathrm{s}\right)$.

Figure $2 b$ is presented to illustrate the much greater variability possible with an orthotropic material compared to the isotropic material illustrated in Fig. 2a. In Fig. 2b, the two longitudinal wave modes are shown propagating along all three axes of symmetry of an orthotropic bone sample. Anisotropy is characterized by three distinct principal values of fabric, $F_{1}, F_{2}$ and $F_{3}$, and their associated perpendicular directions. In Fig. $2 b$ note the variability of the fast wave for porosities lower than about $80 \%$ porosity for the three different directions, as well as in the variability of the slow wave at porosities higher than $80 \%$, for the three directions. In contrast, the slow wave velocity below $80 \%$ porosity and fast wave velocity above $80 \%$, are practically insensitive to the anisotropy of the trabecular bone structure.

These theoretical results indicate that changes in both porosity and anisotropy are mainly shown in the fast wave velocity at low and mild porosities, while these changes are observed in the slow wave velocity mainly at high porosities. In contrast, the slow wave at low and mild porosities is slightly sensitive to changes in porosity and practically insensitive to bone anisotropy; and the fast wave at high porosities (b) Phase Velocity along the axes of symmetry - anisotropy

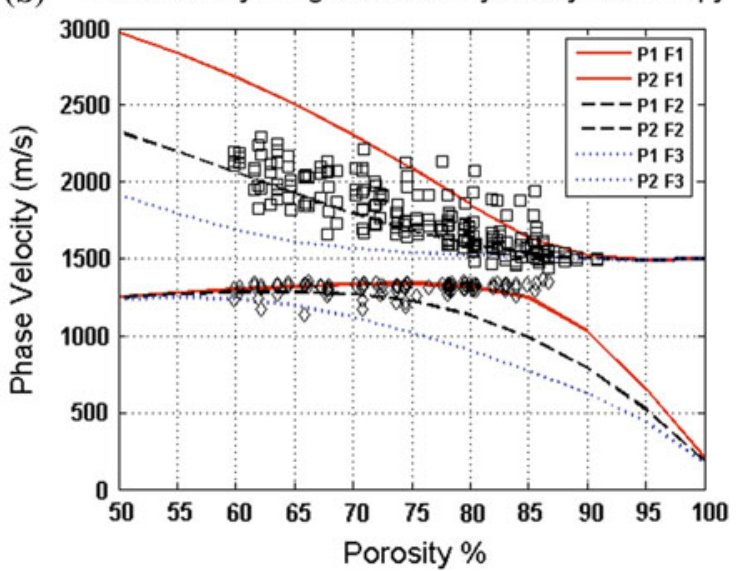

in three orthogonal directions on each sample plotted as a function of the measured bone porosity, and comparison with the theoretical fast (solid) and slow wave velocities (dashed) in anisotropic bone $\left(F_{1}=1.05, F_{2}=1.1, F_{3}=1.15\right)$

is independent of both porosity and anisotropy. All together, these findings indicate the existence of a wave mode transition between the longitudinal wave mode (fast or slow) that is most sensitive to changes in porosity and anisotropy.

Predictions of the fast and slow wave velocities done by this model will now be compared with experimental observations made previously by our group (Cardoso et al. 2003). Briefly, fourteen bovine and sixty human trabecular bone samples were retrieved from bovine femoral heads, human femoral heads and femoral and tibial condyles. Ultrasound wave propagation measurements were obtained from the three orthogonal directions of these cubic shaped samples $(\mathrm{A}, \mathrm{B}$, and $\mathrm{C})$, thus taking into consideration the directional variability of the bone sample microarchitecture. Measurements were performed in immersion with distilled water at room temperature, using two broadband ultrasound transducers (Panametrics V323-SU) at a central frequency of $2.25 \mathrm{MHz}$ ( 0.25 in diameter). The emitter was excited by a damped single pulse generated by an ultrasonic source (Panametrics 5052 UA) operated in a transmission mode. The signal was amplified in $40 \mathrm{~dB}$ and digitized by a $100 \mathrm{MHz}$ Digital Oscilloscope (Tektronic model 2,430) with a digitizing resolution of 10 bits and using a time window of $20.48 \mu \mathrm{s}$, and the post treatment data was performed in MATLAB.

Measurements of wave velocities obtained on three orthogonal directions on each sample were averaged and analyzed as a function of the porosity only (Fig. 3a). Figure $3 b$ shows both the theoretical predictions and experimental wave velocity measurements obtained on three orthogonal directions on each sample. The theoretical curves were computed for fabric anisotropy values $F_{1}, F_{2}$ and $F_{3}$ equal to 1.05 , 1.10 and 1.15 , respectively. This choice of fabric anisotropy 

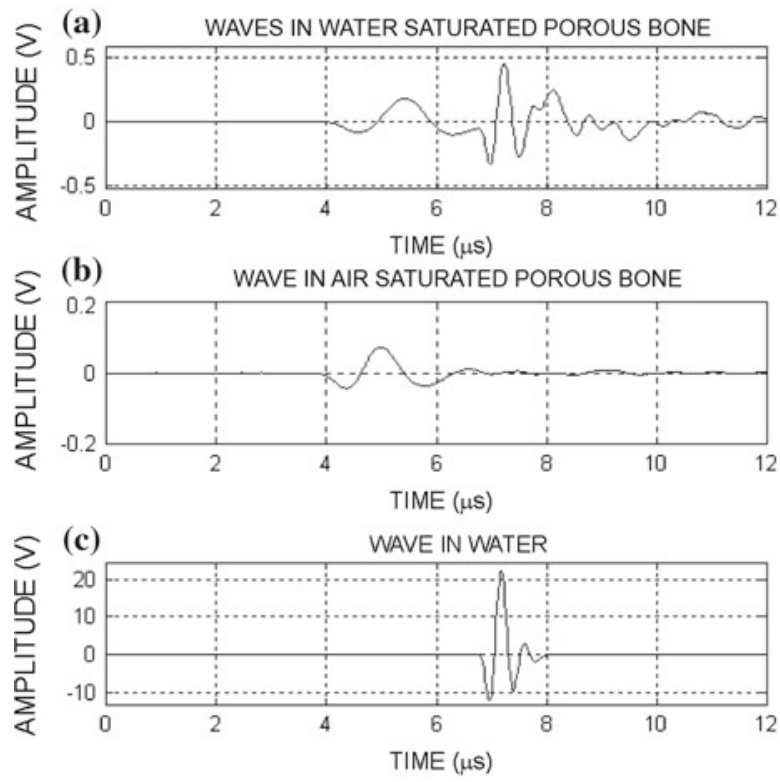

Fig. 4 a Ultrasound wave after propagation through a fluid saturated human cancellous bone sample b signal propagated through the same human sample after the water was removed from the pores, and c detected pulse after propagating in water on a distance identical to the sample's size. Corresponding spectrograms of a human signal showing

corresponds to the degree of anisotropy (5-17\%) measured in the whole set of cancellous bone samples in our previous study (Cardoso et al. 2003). Comparison of experimental data and theoretical results shows a qualitative agreement for both fast and slow wave measurements and these theoretical bounds. However, this last analysis is limited by the fact that the experimental data was obtained measuring the wave propagation in samples that were not cut aligned to their axes of symmetry. Therefore, the measured waves on those samples are not pure wave modes, but quasi-waves. The development of the fabric-dependent anisotropic theory of propagation of quasi-waves in porous media will be presented in a separate study shortly, and a quantitative analysis of these experimental results would be performed.

Another important observation in our previous study of wave propagation in cancellous bone indicated that the fast wave is mostly related to the propagation in the solid structure and the slow wave is highly related to the fluid constituent (Cardoso et al. 2003). Figure 4 shows a typical set of signals obtained in a single direction of a human sample: (i) a well- defined, single ultrasound wave excited the sample (Fig. 4a), (ii) the signal received after propagating through the fluid-saturated cancellous bone sample (Fig. 4b), (iii) the signal received through the sample when the water medium was removed (Fig. 4c) and (iv) the signal received when the cancellous bone sample was removed, thus representing the propagation through the fluid alone (Fig. 4d). From these figures, it is clear that propagation through the cancellous
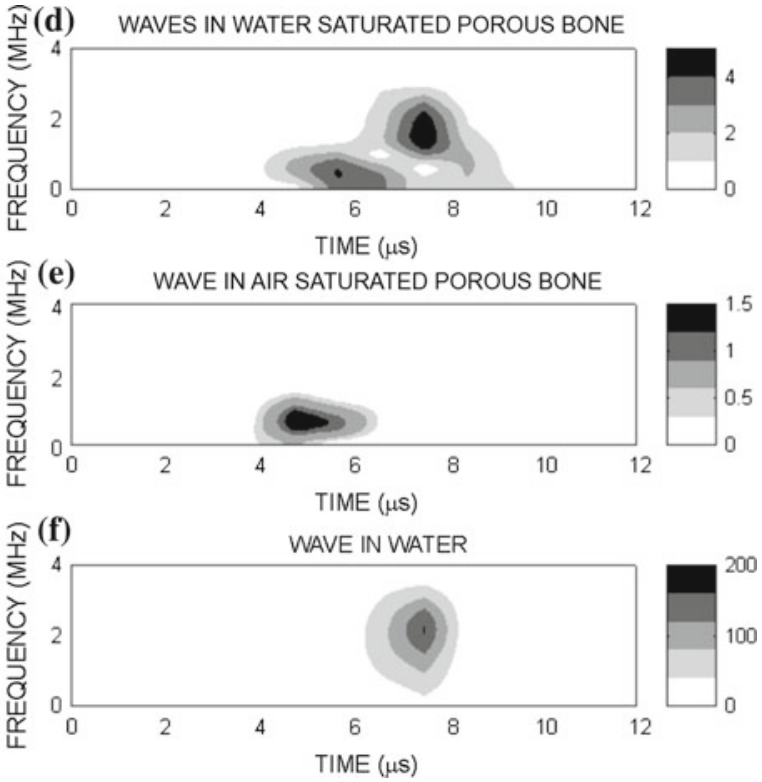

the two waves having different frequency compounds and time localization $\mathbf{d}$, when the fluid is removed from the pores $\mathbf{e}$ and when the porous sample is removed and the wave propagates in the fluid only f. The color bar indicates the respective power spectra density value $\left(\mathrm{Vrms}^{2}\right)$

bone structure dramatically alters the waveform, which after propagation is made of at least two distinguishable waves. When removing the water from the sample (Fig. 4c), only the very first part of the signal remains. On the contrary, when removing the sample while leaving the transducers in place, this first signal disappears and the remaining signal is very similar to the second part of the transmitted signal of Fig. 4b. From these results, one may conclude that the two waves observed with fluid-saturated cancellous bone correspond in general to: (i) a first propagation mode related to the presence of a solid phase within the biphasic material and (ii) a second wave highly related to the effect of the fluid phase.

This observation is also verified by analyzing the wave propagation in the theoretical model when $K_{f}$ tends to 0 . Figure 5 shows the fast and slow wave velocities when the compressibility of the fluid tends to zero, for the isotropic (Fig. 5a) and the anisotropic case (Fig. 5b). Here, the fluid does no longer contribute in the propagation of the waves; thus, the fast wave velocity is equivalent to the wave propagation velocity in the porous solid structure without fluid (as in Fig. 4c), and the slow wave does not propagate.

We return to the consideration of the wave mode transition that occurs for the fast wave at porosities below about $80 \%$ and it is related to the propagation in the solid bony structure, while the slow wave is mostly related to the fluid saturating the pores, as illustrated in Figs. 2 and 3. The behavior of fast and slow waves above about $80 \%$ porosity follows the opposite trend, indicating that the fast wave is mostly related to the 


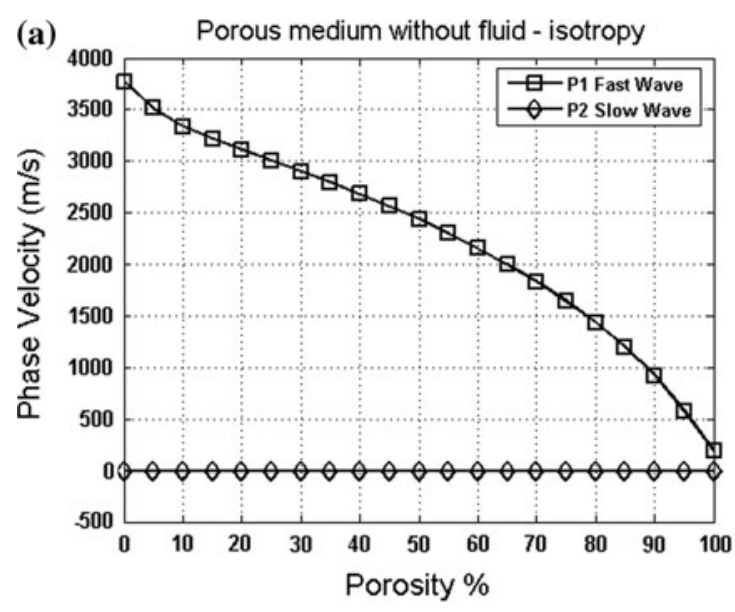

Fig. 5 Phase velocity as a function of porosity of the fast and slow wave modes in isotropic bone medium (a), and along the axes of symmetry in orthotropic bone sample (b) when the compressibility bulk

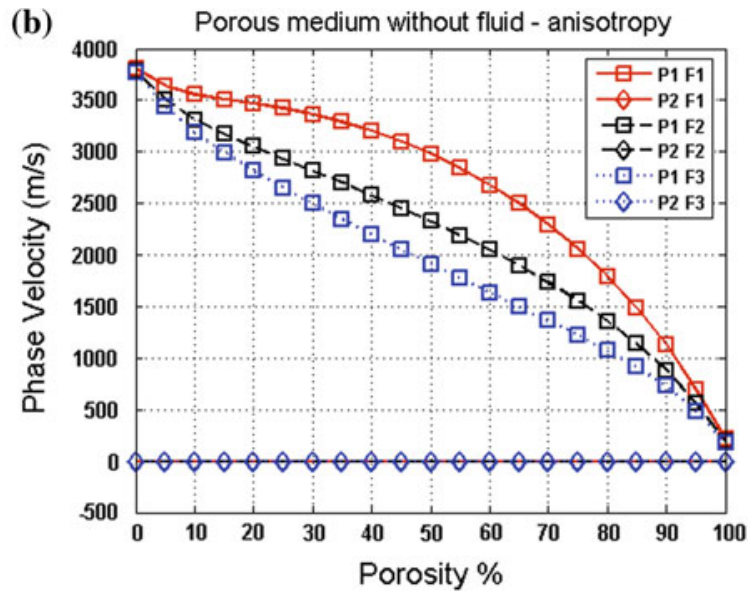

modulus of the fluid is considered equal to zero. Under this condition the fluid does not contribute to the wave propagation
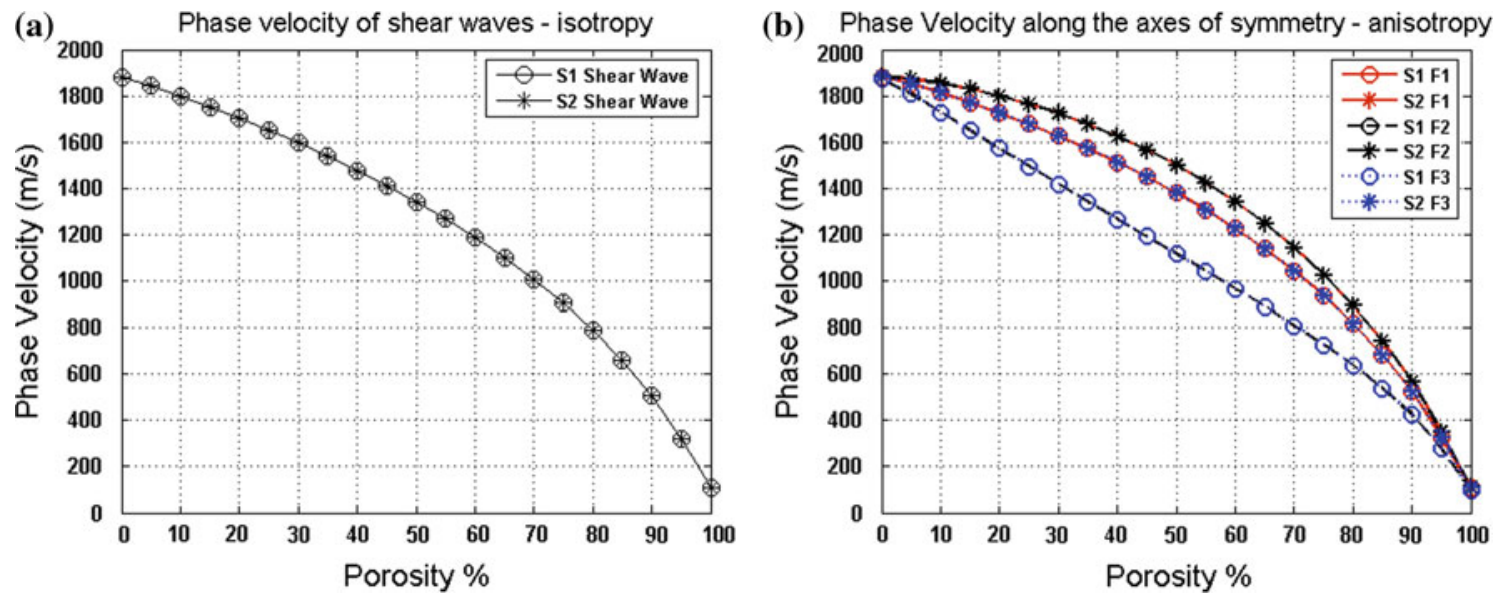

Fig. 6 Phase velocity as a function of porosity of the two shear wave modes in isotropic bone medium (a), and along the axes of symmetry in orthotropic bone samples (b)

propagation in the fluid, and the slow wave is related to the solid bony structure. Both experimental measurements and theoretical predictions presented in this study indicate that the fast wave, when propagating in highly porous samples, is insensitive to the anisotropy of the cancellous bone structure and corresponds to the propagation in the fluid within the pores. The clinical relevance of this finding is that the measurement of the fast wave, the wave measured by most clinical densitometers, lacks sensitivity to provide information on the bone structure when bone becomes osteoporotic.

In addition to the longitudinal waves, the shear waves predicted by the theoretical model were analyzed. The two shear waves S1 (circles) and S2 (stars) have the exact same velocity in the isotropic case (Fig. 2a) and demonstrate a linear dependence on the media's porosity. The two shear waves in the orthotropic medium exhibit a dependence on the bone's anisotropy, but smaller than the one observed in the fast or slow wave velocities (Fig. 6). However, the decrease in the S1 and $\mathrm{S} 2$ wave velocities as a function of porosity is monotonic within the whole range of porosity, and the bone structure anisotropy can be observed as shear wave velocity differences for the three analyzed directions $\left(F_{1}, F_{2}\right.$ and $\left.F_{3}\right)$. Since shear waves cannot propagate in the fluid, $\mathrm{S} 1$ and $\mathrm{S} 2$ velocities do not exhibit the change in behavior at about $80 \%$ porosity that is observed in the $\mathrm{P} 1$ and $\mathrm{P} 2$ longitudinal waves (Fig. 6).

\subsection{Wave dispersion as a function of frequency}

Figure 7 shows both the fast and slow wave velocities as a function of frequency for different values of porosity (Fig. 7a, c, e) and fabric (Fig. 7b, d, f). In Fig. 7a, the acoustic dispersion of all four wave-modes is depicted for a $50 \%$ porosity 

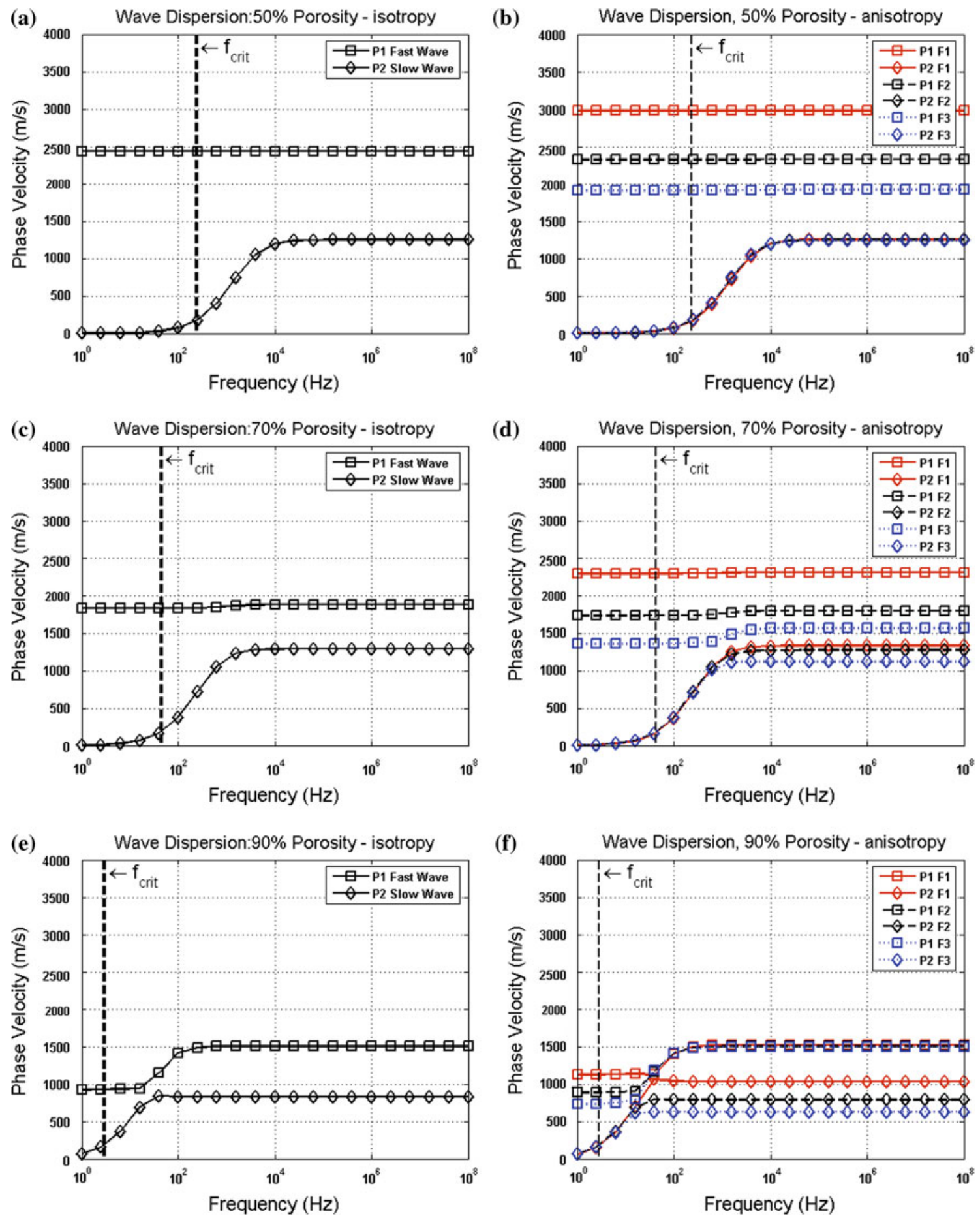

Fig. 7 Wave dispersion of fast and slow longitudinal modes in an isotropic porous media with 50\% (a), $70 \%$ (c) and 90\% porosity (e); as well as in an anisotropic media at $50 \%$ (b), $70 \%$ (d) and $90 \%$ porosity (f)

isotropic bone sample. A strong positive dispersion (velocity increasing with frequency) behavior is observed below the critical frequency in the slow wave. The dispersion of the fast wave is practically negligible at this porosity level, and the velocity of both fast and slow waves is constant at frequencies higher than the critical frequency. Figure $7 \mathrm{c}$ depicts the wave dispersion in an isotropic bone sample with $70 \%$ porosity. This figure shows tendencies that are similar to the ones shown in Fig. 7a, but differ in that the velocities of the fast and shear waves are smaller than the wave velocities in the $50 \%$ porosity medium. Also, a slight dispersion in the fast wave velocity can be observed around the critical frequency 
value, and the slow wave transition from zero to a constant velocity value occurs around the same frequency. Figure 7e, for a $90 \%$ porosity isotropic medium, demonstrates that in a highly porous medium, both slow and fast waves are strongly dispersive. Even more interesting, there also exists a wave mode transition between the slow and the fast wave occurring around the critical frequency. The slow wave has a zero velocity at low frequencies (does not propagate) and above zero it starts propagating with a low speed that increases with the frequency. However, this dispersive behavior of the slow wave changes drastically and its velocity becomes constant for higher frequencies (the curve with diamonds). The fast wave, in turn, has the opposite behavior. It has a constant velocity at low frequencies and a sharp change in dispersion occurs at exactly the same frequency the slow wave dispersion changes. Moreover it must be noted that, in all cases, the slow wave velocity tends to zero as the frequency of the wave approaches zero. At frequencies much higher than the critical frequency, the fast wave has a constant velocity, which is in fact equal to the velocity in the fluid phase of the porous medium, in this case $1480-1500 \mathrm{~m} / \mathrm{s}$.

The observations characterized by diamonds in Fig. 7a,c and e are also distinguished in Fig. 7b,d and f, with the difference that these last include the role of anisotropy. For each wave mode, a set of three curves is produced; the three curves represent the wave propagation along the dynamic axes of symmetry in an orthotropic bone sample $\left(F_{1}, F_{2}\right.$ and $F_{3}$ ). In Fig. $7 \mathrm{~b}$, one can distinguish that the fast and shear waves exhibit changes in their respective velocities as a consequence of the anisotropy. Anisotropy has a mild effect on the slow wave velocity when the porosity is $50 \%$. The effect of the anisotropy on the fast and shear waves is smaller when the porosity increases (Fig. 7e), and a little more pronounced in the slow wave than before. Figure 7f, which corresponds to a $90 \%$ porosity anisotropic medium, shows again a wave mode transition between the fast and slow waves. The fast wave is non-dispersive and sensitive to the anisotropy at low frequencies, while the slow wave becomes the non-dispersive wave mode and sensitive to anisotropy at high frequencies. After the critical frequency, the fast wave becomes dispersive until it reaches the velocity of propagation of sound in the fluid. In contrast to longitudinal waves, the shear waves are non-dispersive but sensitive to the medium's anisotropy.

The critical frequency $f_{\text {crit }}$ changes with both the porosity and the fabric anisotropy. This result demonstrates that the transition in the wave mode from non-dispersive to dispersive is an indicator of the porosity in the media. The critical frequency, and thus the dispersive/non-dispersive behavior of longitudinal waves, also changes for the three analyzed directions in the anisotropic cancellous bone. In theory, above the critical frequency $f_{\text {crit }}=\mu / \pi \rho^{f} d^{2}$, both fast and slow waves may be expected to propagate.
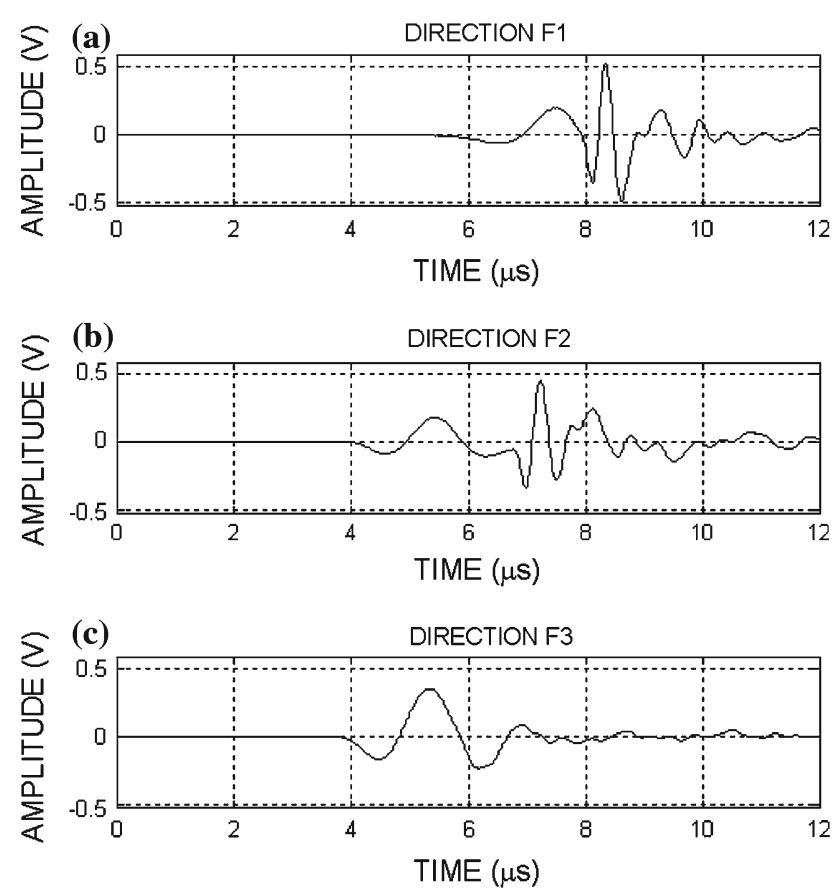

Fig. 8 Ultrasound waves propagated in three orthogonal directions of the same specimen. Only one wave was observed in the third direction. This observation is related to a high attenuation of the slow wave in this direction

Overall, this analysis demonstrates that the acoustic dispersion and the transition between the fast and slow wave modes depend on both the porosity and the fabric anisotropy. These structural parameters and the viscosity of the fluid determine the magnitude of the viscous friction between the solid and the fluid constituents, and thus the frequency in which the transition between the low and high frequency domains of Biot's theory occurs.

The theoretical results shown in Figs. 2, 3 and 7 could explain our experimental observations that the slow wave may not be observed at the three orthogonal directions (A, $\mathrm{B}$ and $\mathrm{C}$ ) of the same specimen. We have hypothesized (Cardoso et al. 2003) that two reasons could explain this observation: (i) the two waves superimpose in the time domain or (ii) the amplitude of the fast and slow waves are very different and settings of the electronics did not allow observing both of them simultaneously. Superimposition of the fast and slow waves was clearly observed in some of the samples, as shown in Fig. 8: in the B direction both waves were observed and could be easily distinguished, while in the A direction, the two waves were found closer to each other and pulses difficult to isolate. This behavior of wave superposition was also observed in Hosokawa's work (Hosokawa and Otani 1998) when changing the ultrasonic propagation direction within the sample. The anisotropic theoretical model predicts specific conditions of porosity and fabric for which the velocities of the fast and 

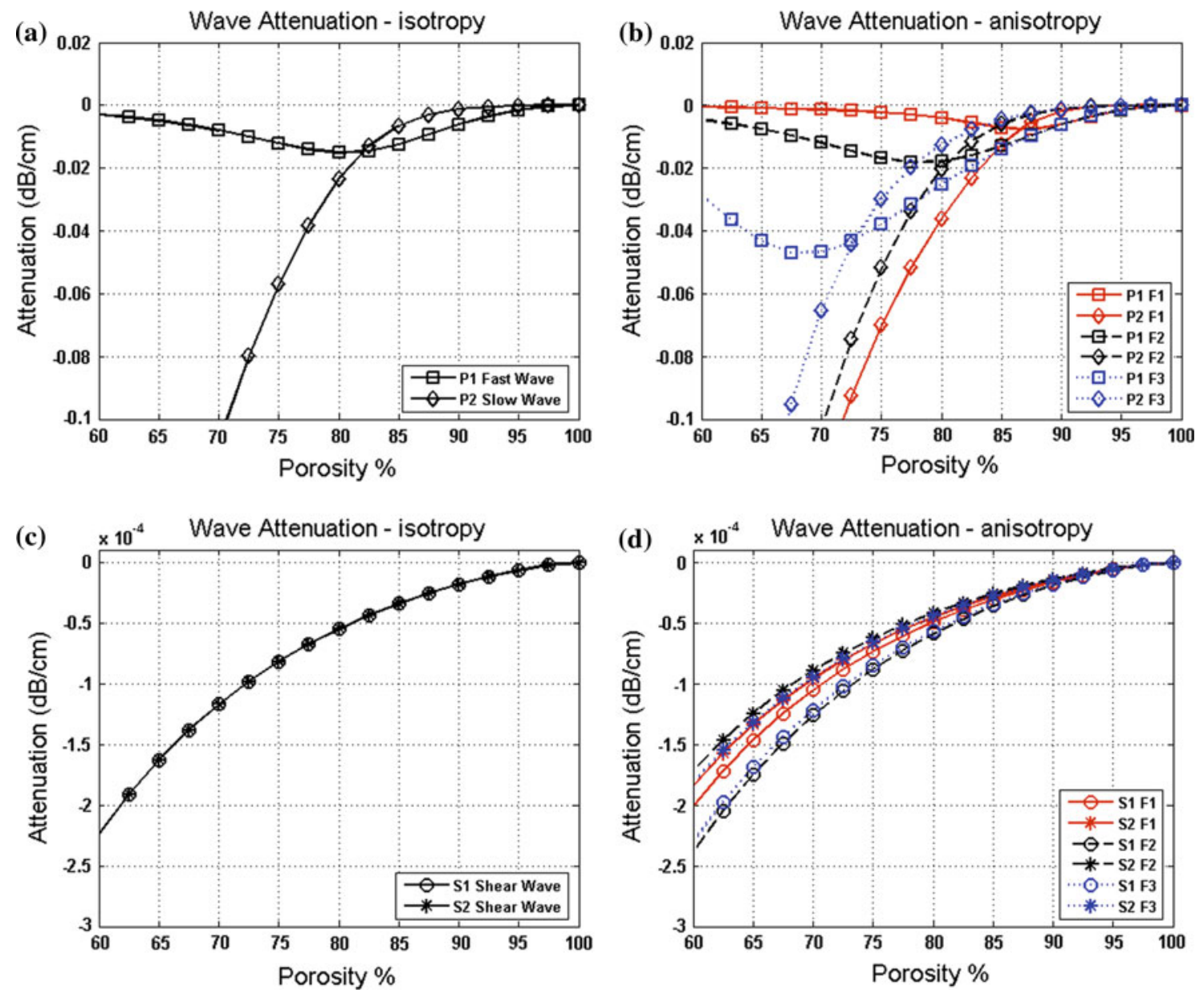

Fig. 9 Attenuation of longitudinal (a and $\mathbf{b})$ and shear waves ( $\mathbf{c}$ and $\mathbf{d}$ ) as a function of porosity in isotropic bone media (a and $\mathbf{c}$ ), and along the axes of symmetry $\left(F_{1}, F_{2}\right.$ and $\left.F_{3}\right)$ in orthotropic bone sample (b and $\mathbf{d}$ )

slow wave modes are almost identical, thus supporting the first interpretation.

6.6 Wave attenuation as a function of porosity or frequency

The ultrasonic attenuation coefficient $\alpha$ represents the amount of energy lost by the ultrasonic beam during its propagation through the medium due to absorption. In porous media, the solution of the poroelastic Christoffel equation gives complex roots since absorption is considered in the model. The complex wave number defines the attenuation coefficient $\alpha$ for the corresponding wave mode

$$
\begin{aligned}
& k_{R e}+i \alpha_{I m}=\frac{\omega}{v_{R e}+i v_{I m}} \\
&=\frac{\omega v_{R e}}{\left(v_{R e}\right)^{2}+\left(v_{I m}\right)^{2}}-i \frac{\omega v_{I m}}{\left(v_{R e}\right)^{2}+\left(v_{I m}\right)^{2}} \\
& \alpha_{I m}=-\frac{\omega v_{I m}}{\left(v_{R e}\right)^{2}+\left(v_{I m}\right)^{2}}
\end{aligned}
$$

and

$\operatorname{Att}_{d B}(\omega)=-20 \log (e)\left(x_{2}-x_{1}\right) \alpha_{I m}$,
The attenuation of longitudinal waves as a function of porosity is shown in Fig. 9a for isotropic, and in 9c for an anisotropic bone sample of $1 \mathrm{~cm}$ size $\left(x_{2}-x_{1}=1 \mathrm{~cm}\right)$ calculated at $1 \mathrm{MHz}$. Attenuation of the fast wave (squares) is smaller than the attenuation of the slow wave at porosities below about $80 \%$. However, the attenuation of both waves change in behavior above about $80 \%$, and the slow wave becomes slightly less attenuated than the fast wave (Fig. 9a). The porosity at which this transition between fast and slow wave occurs is, however, affected by the fabric anisotropy, as shown in Fig. 9b. This observation indicates that there exist a range of porosity and anisotropy where the absorption-related attenuation for both waves is of the same order or magnitude. Therefore, whether both waves may have similar amplitude and might be observed simultaneously depends on both the porosity and anisotropy of the sample.

In addition to the role of the porosity and fabric anisotropy, the dependence of attenuation on frequency was analyzed. The attenuation of longitudinal waves on frequency is shown in Fig. 10a for isotropic, and in 10b for an anisotropic bone sample of $1 \mathrm{~cm}$ size and $80 \%$ porosity. Attenuation of 

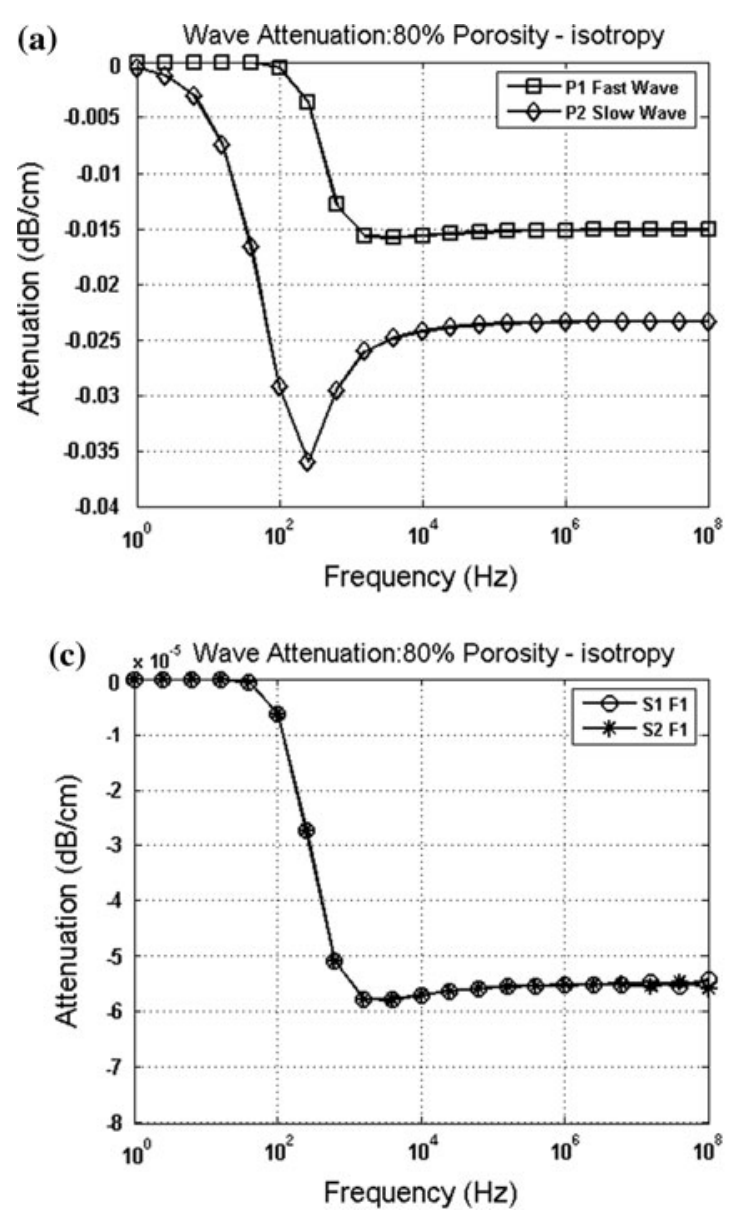

Fig. 10 Wave attenuation as a function of frequency of the fast and slow wave modes in isotropic bone medium (a), along the axes of symmetry $\left(F_{1}, F_{2}\right.$ and $\left.F_{3}\right)$ in orthotropic bone sample $(\mathbf{b})$, shear wave modes

the fast wave (squares) is smaller than the attenuation of the slow wave at low frequencies. However, the attenuation of both waves change in behavior, and the slow wave becomes slightly less attenuated than the fast wave at high frequencies (Fig. 10a). The frequency at which this transition between fast and slow wave occurs is determined by the pore diameter, fluid density and viscosity. Figure 10b shows that the transition in attenuation between the fast and slow wave also depends on the fabric. The transition in attenuation between the fast and slow waves only occurs at direction $F_{1}$, but not in $F_{2}$ and $F_{3}$, for which the slow wave remains the most attenuated for all frequencies.

The implication of these results is that both the porosity and the fabric play a critical role in the absorption-dependent attenuation of longitudinal waves. This theoretical result is also a plausible explanation for the observation of two waves with a high difference in their respective amplitude. Figure 11 provides a signal obtained with a highly porous sample where the fast wave is almost undetectable. This fast wave still existed, as demonstrated in Fig. 11b where the signal
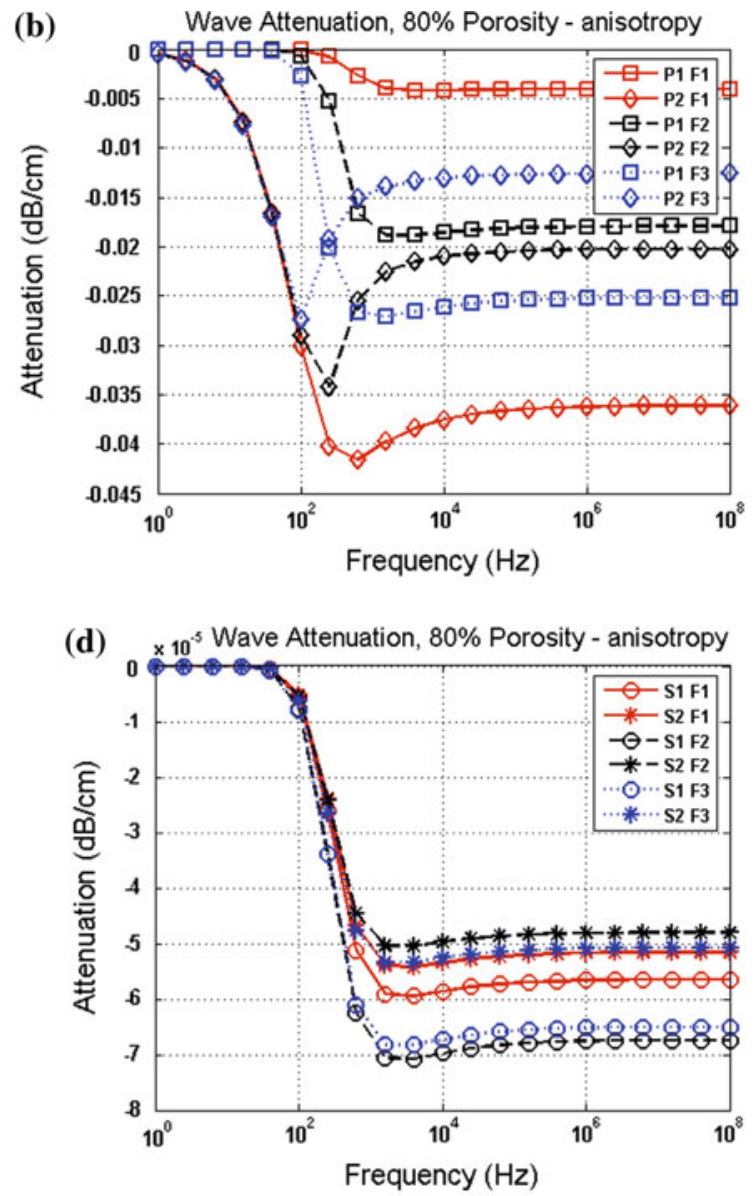

in isotropic bone medium (c), and shear waves along the axes of symmetry $\left(F_{1}, F_{2}\right.$ and $\left.F_{3}\right)$ in orthotropic bone sample $(\mathbf{d})$

was magnified, and its amplitude was about 30 times lower than that of the slow wave. The theoretical results shown in Fig. 9 indicate that the fast wave can be more attenuated than the slow wave at certain conditions of porosity and fabric, and could explain our experimental observations that one of the two waves may be overlooked and remain unmeasured. In Fig. 11, the undetected wave is the fast mode, and in Fig. 8c is the slow wave. Superimposition of the fast and slow waves was thus clearly predicted by the anisotropic poroelastic model.

\section{Discussion}

Anisotropic poroelastic wave propagation theory was extended in this study by introducing the dependence of the wave motion equations upon fabric, a tensorial descriptor of the porous microarchitecture. Solution of the constitutive equations for harmonic displacements of the solid and fluid constituents leads to a modified Christoffel equation for anisotropic porous media that includes the acoustic tensor 

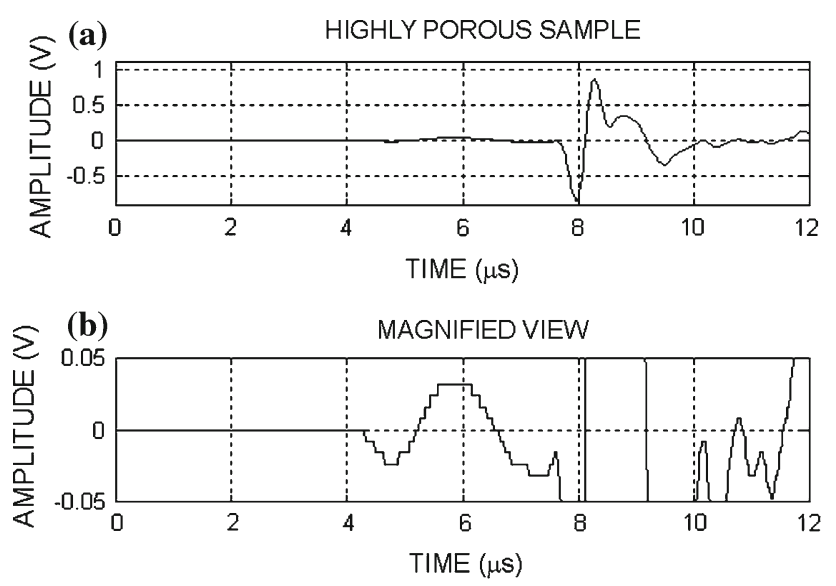

Fig. 11 Case of signals detected in a very porous sample where (a) the fast wave was almost imperceptible. $\mathbf{b}$ a higher magnification showing the existence of the fast wave

$\mathbf{Q}$, the solid-fluid interaction tensor $\mathbf{C}$, and the permeability tensor $\mathbf{K}(\omega)$. These tensors describe the elastic and viscous effects in the wave equation, and they all depend on the measurable fabric tensor, $\mathbf{F}$. The modified Christoffel equation represents an eigenvalue problem with a sixth order characteristic equation and four non-zero roots. This system reduces to the isotropic formulation developed by Biot when the fabric tensor is isotropic. Two eigenvalues represent the longitudinal wave modes $\mathrm{P} 1$ and $\mathrm{P} 2$ and the other two correspond to the shear wave modes $\mathrm{S} 1$ and $\mathrm{S} 2$. Such eigenvalues are complex valued, and describe the phase velocity and attenuation due to absorption of the four wave modes.

Propagation of plane waves in both isotropic and anisotropic saturated porous media was analyzed as a function of the porosity and fabric. Elastic constant and density values for the mineralized bone tissue and water were used in the poroelastic model to study the wave propagation in cancellous bone. Two constants are used to describe the solid phase $\left(E^{\mathrm{s}}\right.$ and $\left.\rho^{\mathrm{s}}\right)$, three for the fluid phase $\left(\rho^{\mathrm{f}}, K^{\mathrm{f}}\right.$ and $\left.\mu\right)$ and one constant to relate the porosity to the pore diameter. Two independent variables ( $\phi$ and $\mathbf{F}$ ), one scalar and the other tensorial, respectively, were integrated in the model to study the influence of material properties on both global and directional changes in the velocity and attenuation of the four wave modes generated in porous media.

The theoretical model predicted that in isotropic media with porosity below $80 \%$, the fast wave decreases with the porosity. At porosities higher than $80 \%$, the fast wave exhibits a constant velocity, and the slow wave is the wave mode that is sensitive to changes in porosity. This result demonstrated that one of the two wave modes is more sensitive than the other to changes in porosity; however, there exists a transition in sensitivity between the two longitudinal wave modes at approximately $80 \%$ porosity. The fast wave is sensitive to the porosity when the apparent modulus to density ratio of the solid phase $\left((1-\phi) K^{\mathrm{s}} / \rho^{\mathrm{s}}\right)$ is higher than that of the fluid phase $\left(\phi K^{\mathrm{f}} / \rho^{\mathrm{f}}\right)$; while the slow wave is sensitive to porosity when the apparent modulus to density ratio of the solid phase is smaller than that of the fluid phase. Therefore, the porosity level at which this transition between the fast and the slow wave modes occurs is a consequence of the intrinsic properties of the solid $\left(E^{\mathrm{S}}\right.$ and $\left.\rho^{\mathrm{S}}\right)$ and fluid $\left(\rho^{\mathrm{f}}, K^{\mathrm{f}}\right.$ and $\mu$ ) constituents. For instance, if the properties for the fluid phase are changed to those of glycerol $\left(\rho^{\mathrm{f}}=\right.$ $1,261 \mathrm{Kg} / \mathrm{m}^{3}, K^{\mathrm{s}}=4.35 \mathrm{GPa}$, and $\mu=1.5 \mathrm{Pas}$ ), the transition between the wave modes happens at $65 \%$ and with ethanol at $20^{\circ} \mathrm{C}\left(\rho^{\mathrm{f}}=789 \mathrm{Kg} / \mathrm{m}^{3}, K^{\mathrm{f}}=0.902 \mathrm{GPa}\right.$, and $\mu=1.2 \times 10^{-3} \mathrm{Pas}$ ) the transition between the wave modes happens at $90 \%$ (figure not shown). The shear wave modes, in contrast, are not affected by the presence of the fluid and do not exhibit a change in behavior as shown in the longitudinal waves. Shear waves are, however, sensitive to both porosity and anisotropy.

It is important to note that the porosity at which the fast wave - slow wave mode transition occurs in longitudinal waves depends on the fabric anisotropy. The theoretical model predicted that mild changes in anisotropy would produce this transition to occur in the porosity range between 70 and $90 \%$. In an orthotropic bone sample, the direction with lower modulus will exhibit a transition at a lower porosity than the direction with intermediate and high modulus. Consequently, this theoretical model indicates that an anisotropic bone sample with approximately $80 \%$ porosity may exhibit either a fast or slow wave that is sensitive or insensitive to the anisotropy of the medium depending on the direction being analyzed.

The wave mode transition observed in the analysis of velocity as a function of porosity and fabric anisotropy is also observed when the wave velocity is analyzed as a function of the frequency. The wave mode transition occurs around the critical frequency in samples with high porosity $(>80 \%)$. The fast and slow wave dispersion changes drastically in behavior at the frequency where the velocity (and the wave length) of both wave modes becomes very similar. However, the frequency at which this transition occurs is also affected by the fabric anisotropy. Therefore, the transition frequency from being non-dispersive to dispersive and vice versa is a consequence of the intrinsic and extrinsic properties of the medium (material constituents, porosity and fabric anisotropy). The transition between the low and high frequency regimes defined by Biot (critical frequency) at which the slow wave becomes a propagative wave mode occurs at frequencies much lower than the ones usually employed for ultrasound characterization of bone. In fact, the model predicted very low acoustic dispersion into the range of porosity, fabric and frequencies generally used in clinical applications. It is important to notice that the wave dispersion predicted by the model is a consequence of the absorption in either iso- 
tropic media or in anisotropic media along the dynamic axes of the sample. However, dispersion processes may be more complex when the wave propagation is analyzed in directions that are not normal to planes of material symmetry.

The theoretical model also predicted the high variability of fast and slow wave velocities observed in bovine and human bone in our experimental study. Comparison of experimental data and theoretical results shows a qualitative agreement for both fast and slow wave velocities. Directional variability within a sample was effectively explained by the theoretical model after inclusion of the fabric; this directional variability could not be explained by the porosity only. The agreement between experimental and theoretical values in this study indicate that despite the complexity added to the poroelastic theory, a tensorial variable describing the bone microstructure is required to explain the directional variability of the wave propagation with bone architecture. Nonetheless, the comparison between experiments and theoretical predictions in this study is limited by the fact that the experimental data was obtained measuring the wave propagation in samples that were not cut aligned to their axes of symmetry. Therefore, the measured waves on those samples are not pure wave modes, but quasi-waves. The development of the fabric-dependent anisotropic theory of propagation of quasiwaves in porous media will be presented in a separate study, and a quantitative analysis of these experimental results will be performed. Moreover, solid and fluid interaction phenomena should be thoroughly investigated. For this reason, studies of ultrasonic wave propagation properties using various solid porous materials mimicking cancellous bone structure as well as various saturating fluids exhibiting different physical properties (elasticity, density, viscosity) are needed.

The analysis of the wave attenuation as a function of the porosity, fabric and frequency demonstrated that there exist a range of porosity and anisotropy - at a given fixed frequency - in which the attenuation due to absorption is of the same order of magnitude for both fast and slow wave modes. Similarly to the longitudinal wave velocities, a transition in the attenuation of the two wave modes occurs around $80 \%$ in an isotropic medium and within the $70-90 \%$ range when anisotropy is considered. The important implication of this theoretical result is that, depending on the porosity and the fabric anisotropy of the sample, one wave mode-either the fast or the slow-may be highly attenuated with respect to the other and remain practically undetected as shown in our experimental study. Notice that the fast to slow wave attenuation ratio depends on the porosity and fabric anisotropy. In other words, the direction in which the sample is interrogated may not allow a clear observation of both waves simultaneously if they superimpose due to having similar velocities or having very different attenuations. This may explain why the clinical densitometer systems measuring the wave propagation in the medial-lateral direction at the calca- neum might not be able to distinguish, thus far, both waves in vivo. Usually, velocity and attenuation measurement methods (in clinical densitometers) presuppose that only one wave propagates in cancellous bone. However, if only one wave is observable/measurable at a given direction, then it is even more important to distinguish whether that wave is the fast or the slow wave mode, and whether that wave mode is sensitive or not to the anisotropy of the cancellous porous structure.

Overall, the results from the present study demonstrate the ability of the proposed model to describe the acoustic behavior of the fast and slow wave velocities in cancellous bone. Both phase velocity and attenuation are dependent on the architecture (porosity and fabric) and the composition of the medium (solid and fluid mass density, solid elastic modulus, fluid bulk modulus and fluid viscosity). For given frequency and material parameter values, the behaviors of the fast and slow waves are governed by the extrinsic properties of the media: the porosity and fabric anisotropy. These theoretical predictions also corroborate our experimental observations which indicate that at high porosities the fast wave is mostly related to the propagation in the fluid constituent and the slow wave is highly related to the solid structure. Therefore, the theoretical predictions confirm our observations that the measurement of the fast wave lacks sensitivity to provide information on the bone structure when bone becomes osteoporotic. In contrast to empirical relationships used by ultrasound densitometers, the fabric-dependent anisotropic poroelastic model proposed in this study provides a theoretical framework to predict, analyze and interpret changes in elastic constants of the trabecular bone structure. Since the velocity and attenuation predictions provided by this novel approach depend on the tissue composition, porosity and architecture of the cancellous bone sample, it has the potential to characterize bone quality beyond BMD.

Acknowledgments This work was supported by the National Institutes of Health (AG34198 \& HL069537-07 R25 Grant for Minority BME Education), the National Science Foundation (NSF 0723027, PHY-0848491), and the PSC-CUNY Research Award Program of the City University of New York.

Open Access This article is distributed under the terms of the Creative Commons Attribution Noncommercial License which permits any noncommercial use, distribution, and reproduction in any medium, provided the original author(s) and source are credited.

\section{Appendix}

The purpose of this appendix is to record the derivation of the formulas for the three Young's moduli and the three shear moduli expressed in terms of the porosity $\phi$ and the principal values of the fabric $F_{i}, i=1,2,3$ in Eqs. (38) and (39). These results stem from the dependence of the compliance tensor upon fabric is given by [Eq. 4 in Cowin (1985)]: 


$$
\begin{aligned}
S_{i j k m}= & a_{1} \delta_{i j} \delta_{k m}+a_{2}\left(F_{i j} \delta_{k m}+\delta_{i j} F_{k m}\right) \\
& +a_{3}\left(\delta_{i j} F_{k q} F_{q m}+\delta_{k m} F_{i q} F_{q j}\right) \\
& +b_{1} F_{i j} F_{k m}+b_{2}\left(F_{i j} F_{k q} F_{q m}+F_{k m} F_{i q} F_{q j}\right) \\
& +b_{3} F_{i s} F_{s j} F_{k q} F_{q m}+c_{1}\left(\delta_{k i} \delta_{m j}+\delta_{m i} \delta_{k j}\right) \\
& +c_{2}\left(F_{k i} \delta_{m j}+F_{k j} \delta_{m i}+F_{i m} \delta_{k j}+F_{m j} \delta_{k i}\right) \\
& +c_{3}\left(F_{i r} F_{r k} \delta_{m j}+F_{k r} F_{r j} \delta_{m i}+F_{i r} F_{r m} \delta_{k j}\right. \\
& \left.+F_{m r} F_{r j} \delta_{i k}\right),
\end{aligned}
$$

where the coefficients $a_{1}, a_{2}, a_{3}, b_{1}, b_{2}, b_{3}, c_{1}, c_{2}$ and $c_{3}$ are functions of $\phi$ and the two invariants of $\mathbf{F}$, II and III,

$$
\begin{aligned}
& a_{1}\left(V_{v}, I I, I I I\right)=g_{1}+g_{2}+g_{3}+g_{4} I I, \\
& a_{2}\left(V_{v}, I I, I I I\right)=g_{5}+g_{6} I I, a_{3}\left(V_{v}, I I, I I I\right)=g_{7} I I, \\
& b_{1}\left(V_{v}, I I, I I I\right)=g_{8} I I, b_{2}\left(V_{v}, I I, I I I\right)=0, \\
& b_{3}\left(V_{v}, I I, I I I\right)=0, c_{1}\left(V_{v}, I I, I I I\right)=h_{1}+h_{2}+h_{3}+h_{4} I I \\
& b_{2}\left(V_{v}, I I, I I I\right)=h_{5}+h_{6}, c_{3}\left(V_{v}, I I, I I I\right)=h_{7},
\end{aligned}
$$

and the fabric tensor is normalized by the trace of $\mathrm{F}$ so that $I=\operatorname{tr} \mathbf{F}=1$. Thus the orthotropic elastic constants may be deduced from (A-1) and are given by,

$$
\begin{gathered}
1 / E_{i}=\frac{1}{E_{s}}\left[k_{1}+2 k_{6}+\left(k_{2}+2 k_{7}\right) I I+2\left(k_{3}+2 k_{8}\right) F_{1}\right. \\
\left.+\left(2 k_{4}+k_{5}+4 k_{9}\right) F_{1}^{2}\right], \\
\frac{1}{G_{i j}}=\frac{1}{G_{s}}\left[k_{6}+k_{7} I I+k_{8}\left(F_{i}+F_{j}\right)+k_{9}\left(F_{i}^{2}+F_{j}^{2}\right)\right], \\
v_{i j}=-E_{i}\left[k_{1}+k_{2} I I+k_{3}\left(F_{i}+F_{j}\right)+k_{4}\left(F_{i}^{2}+F_{j}^{2}\right)\right. \\
\left.+k_{5} F_{i} F_{j}\right] .
\end{gathered}
$$

where

$k_{1}=g_{1}+g_{2}+g_{3}, k_{2}=g_{4}, k_{3}=g_{5}+g_{6}, k_{4}=g_{7}, k_{5}=g_{8}$,

$k_{6}=h_{1}+h_{2}+h_{3}, k_{7}=h_{4}, k_{8}=h_{5}+h_{6}, k_{9}=h_{7}$,

$k_{1}$ to $k_{9}$ are compliance coefficients that depend on the volume fraction (Turner et al. 1990). Following a similar development, the dependence of the stiffness tensor upon fabric leads to the following relationships:

$$
\begin{aligned}
& E_{i}=E_{S}\left[m_{1}+m_{2} I I+m_{3} F_{1}+m_{4} F_{1}^{2}\right], \\
& G_{i j}=G_{s}\left[m_{5}+m_{6} I I+m_{7}\left(F_{i}+F_{j}\right)+m_{8}\left(F_{i}^{2}+F_{j}^{2}\right)\right],
\end{aligned}
$$

$$
\begin{aligned}
& v_{i j}=-E_{i} \\
& \times\left[\begin{array}{c}
\left(\frac{1}{m_{1}}-\frac{2}{m_{5}}\right)+\left(\frac{2 m_{6}}{m_{5}^{2}}-\frac{m_{2}}{m_{1}^{2}}\right) I I+\left(\frac{2 m_{7}}{m_{5}^{2}}-\frac{m_{3}}{2 m_{1}^{2}}\right) \\
\left(F_{i}+F_{j}\right) \ldots \\
\ldots+\left\{\frac{1}{3}\left(\frac{m_{3}^{2}}{m_{1}^{3}}-\frac{m_{4}}{m_{1}^{2}}-\frac{4 m_{1} m_{7}^{2}}{m_{5}^{4}}+\frac{4 m_{8}}{m_{1}^{2}}\right)\right\} \\
\left\{\left(F_{i}^{2}+F_{j}^{2}\right)+F_{i} F_{j}\right\}
\end{array}\right] .
\end{aligned}
$$

in which the stiffness coefficients $m_{1}$ to $m_{8}$ are related to $k_{1}$ to $k_{9}$ by

$$
\begin{aligned}
& m_{1}=\frac{1}{\left[k_{1}+2 k_{6}\right]}, m_{2}=-\frac{k_{2}+2 k_{7}}{\left[k_{1}+2 k_{6}\right]^{2}}, m_{3}=-\frac{2\left(k_{3}+2 k_{8}\right)}{\left[k_{1}+2 k_{6}\right]^{2}}, \\
& m_{4}=-\frac{2 k 4+k_{5}+4 k_{9}}{\left[k_{1}+2 k_{6}\right]^{2}}+\frac{4\left[k_{3}+2 k_{8}\right]^{2}}{\left[k_{1}+2 k_{6}\right]^{3}}, \\
& m_{5}=\frac{1}{k_{6}}, m_{6}=-\frac{k_{7}}{k_{6}^{2}}, m_{7}=-\frac{k_{8}}{k_{6}^{2}}, m_{8}=-\frac{k_{9}}{k_{6}^{2}}+\frac{k_{8}^{2}}{k_{6}^{3}}
\end{aligned}
$$

Either $k$ 's or $m$ 's are usually obtained from curve fitting of theory to experimental measurements or finite element modeling. Such approaches have shown a dependence of the stiffness coefficients in the volume fraction to a power $n$ that ranges from 0.5 to 3 , and conversely, the compliance coefficients depend on the volume fraction to the power $-n$. Here, it is proposed that the coefficients $m_{1}$ through $m_{6}$ are functions that depend on the volume fraction following the relationships

$$
\begin{aligned}
& m_{1}=d_{2}\left[(1-\phi)^{n}+d_{1} \phi(1-\phi)^{n}\right], \\
& m_{2}=m_{3}=m_{4}=-d_{1} \phi(1-\phi)^{n} \\
& m_{5}=d_{3}\left[(1-\phi)^{n}+d_{1} \phi(1-\phi)^{n}\right], \\
& m_{6}=m_{7}=m_{8}=-d_{1} \phi(1-\phi)^{n}
\end{aligned}
$$

thus, Eqs. A-7 and A-8 become

$$
\begin{aligned}
& E_{i}=E_{s}\left[d_{2}(1-\phi)^{n}+d_{1} \phi(1-\phi)^{n}\left(d_{2}-I I-F_{i}-F_{i}^{2}\right)\right], \\
& G_{i j}=G_{s}\left[d_{3}(1-\phi)^{n}+d_{1} \phi(1-\phi)^{n}\right. \\
& \left.\quad \times\left[d_{3}-I I-\left(F_{i}+F_{j}\right)_{i}-\left(F_{i}^{2}+F_{j}^{2}\right)\right]\right] .
\end{aligned}
$$

The left term inside the bracket in Eqs. A-13 and A-14 is dependent on the volume fraction and independent of fabric, but the term in the right hand side of Eqs. A-13 and A-14 depend on both volume fraction and fabric. When the porous medium is isotropic, the term in the right hand side is required to vanish. Therefore, the coefficients $d_{2}$ and $d_{3}$ are constants that can be obtained from the requirement of isotropy $F_{i}=F_{j}=1 / 3$. It is important to note that the term in the right hand side of Eqs. A-13 and A-14 represents the deviation from isotropy; the coefficient $d_{1}$ is a proportionality constant between the magnitude of the anisotropy in the porous medium and the fabric components measured using 
the mean intercept length or any other method of determining the fabric,

$$
\begin{aligned}
d_{2} & =I I+F_{i}+F_{i}^{2}=\frac{1}{3}+\frac{1}{3}+\frac{1}{9}=\frac{7}{9}=0.7778 \\
d_{3} & =I I+\left(F_{i}+F_{j}\right)+\left(F_{i}^{2}+F_{j}^{2}\right)=\frac{1}{3}+\frac{2}{3}+\frac{2}{9} \\
& =\frac{11}{9}=1.2222
\end{aligned}
$$

Thus, the coefficients $k_{1}$ to $k_{9}$ are described by

$$
\begin{aligned}
k_{1}= & {\left[\frac{1}{d_{2}}-\frac{2}{d_{3}}\right] \frac{1}{\left[(1-\phi)^{n}+d_{1} \phi(1-\phi)^{n}\right]} } \\
k_{2}= & {\left[\frac{1}{d_{2}^{2}}-\frac{2}{d_{3}^{2}}\right] \frac{d_{1} \phi(1-\phi)^{n}}{\left[(1-\phi)^{n}+d_{1} \phi(1-\phi)^{n}\right]^{2}} } \\
k_{3}= & {\left[\frac{1}{2 d_{2}^{2}}-\frac{2}{d_{3}^{2}}\right] \frac{d_{1} \phi(1-\phi)^{n}}{\left[(1-\phi)^{n}+d_{1} \phi(1-\phi)^{n}\right]^{2}} } \\
k_{4}= & k_{5}=\frac{1}{3}\left\{\left[\frac{1}{C_{2}^{3}}\right]-\left[\frac{4}{d_{3}^{3}}\right]\right\} \frac{\left[d_{1} \phi(1-\phi)^{n}\right]^{2}}{\left[(1-\phi)^{n}+d_{1} \phi(1-\phi)^{n}\right]^{3}} \\
k_{6}= & {\left[\frac{1}{d_{3}}\right] \frac{1}{\left[(1-\phi)^{n}+d_{1} \phi(1-\phi)^{n}\right]} } \\
k_{7}= & {\left[\frac{1}{d_{3}^{2}}\right] \frac{1}{\left[(1-\phi)^{n}+d_{1} \phi(1-\phi)^{n}\right]^{2}} } \\
k_{8}= & {\left.\left.\left[\frac{1}{d_{2}^{2}}\right] \frac{d_{1} \phi(1-\phi)^{n}}{d_{3}^{2}}\right]\right\} \frac{\left.d_{1} \phi(1-\phi)^{n}+d_{1} \phi(1-\phi)^{n}\right]^{2}}{\left[(1-\phi)^{n}+d_{1} \phi(1-\phi)^{n}\right]^{2}} } \\
k_{9}= & {\left[\frac{1}{d_{3}^{3}}\right] \frac{\left[d_{1} \phi(1-\phi)^{n}\right]^{2}}{\left[(1-\phi)^{n}+d_{1} \phi(1-\phi)^{n}\right]^{3}} } \\
+ & \left.\frac{1}{d_{3}^{2}}\right] \frac{d_{1} \phi(1-\phi)^{n}}{\left[(1-\phi)^{n}+d_{1} \phi(1-\phi)^{n}\right]^{2}} .
\end{aligned}
$$

The coefficients $k_{1}^{s d}$ through $k_{9}^{s d}$ appear in the formulas for the three Young's moduli and the three shear moduli (38) and (39). The formulas (A-3) and (A-4) are recorded as Eqs. (38) and (39) in the text.

\section{References}

Ashman RB, Rho JY (1988) Elastic modulus of trabecular bone material. J Biomech 21:77-181

Auld B (1973) Acoustic fields and waves in solids, vol. 1. Wiley, New York

Baroud G, Falk R, Crookshank M, Sponagel S, Steffen T (2004) Experimental and theoretical investigation of directional permeability of human vertebral cancellous bone for cement infiltration. J Biomech 37:189-196

Basillais A, Bensamoun S, Chappard Ch, Brunet-Imbault B, Lemineur G, Ilharreborde B, Ho Ba Tho MC, Benhamou CL (2007) Threedimensional characterization of cortical bone microstructure by microcomputed tomography: validation with ultrasonic and microscopic measurements. J Orthop Sci 12(2):141-148

Bear Jacob (1988) Dynamics of fluids in porous media. Dover Publications Inc., Mineola, p 134

Beaudoin AJ, Mihalko WM, Krause WR (1991) Finite element modelling of polymethylmethacrylate flow through cancellous bone. J Biomech 24(2):127-136

Biot MA (1941) General theory of three-dimensional consolidation. J Appl Phys 12:155-164

Biot MA (1955) Theory of elasticity and consolidation for a porous anisotropic solid. J Appl Phys 26:182-185

Biot MA (1956a) Theory of propagation of elastic waves in a fluid saturated porous solid I low frequency range. J Acoust Soc Am 28:168178

Biot MA (1956b) Theory of propagation of elastic waves in a fluid saturated porous solid II higher frequency range. J Acoust Soc Am 28:179-191

Biot MA (1962a) Mechanics of deformation and acoustic propagation in porous media. J Appl Phys 33:1482-1498

Biot MA (1962b) Generalized theory of acoustic propagation in porous dissipative media. J Acoust Soc Am 28:1254-1264

Bolotin HH (2007) DXA in vivo BMD methodology: an erroneous and misleading research and clinical gauge of bone mineral status, bone fragility, and bone remodeling. Bone 41(1):138-154

Bone HG, Santora AC, Chattopadhyay A, Liberman U (2005) Are we treating women with postmenopausal osteoporosis for their low BMD or high fracture risk? J Bone Miner Res 20:2064-2065

Cardoso L, Meunier A, Oddou C (2008) In vitro acoustic wave propagation in human and bovine cancellous bone as predicted by the Biot's theory. J Mech Med Biol 8(2):1-19

Cardoso L, Teboul F, Meunier A, Oddou C (2001) Ultrasound characterization of cancellous bone: theoretical and experimental analysis. IEEE Trans ultrason Symp 2:1213-1216

Cardoso L, Teboul F, Sedel L, Meunier A, Oddou C (2003) In vitro acoustic waves propagation in human and bovine cancellous bone. J Bone Mineral Res 18(10):1803-1812

Cowin SC, Mehrabadi MM (2007) Compressible and incompressible constituents in anisotropic poroelasticity: the problem of unconfined compression of a disk. J Mech Phys Solids 55:161-193

Cowin SC (2004) Anisotropic poroelasticity: fabric tensor formulation. Mech Mater 36:665-677

Cowin SC, Satake M (eds) (1978) Continuum mechanical and statistical approaches in the mechanics of granular materials. Gakujutsu Bunken Fukyu-Kai, Tokyo

Cowin SC (1999) Bone poroelasticity. J Biomech 32:218-238

Cowin SC (1985) The relationship between the elasticity tensor and the fabric tensor. Mech Mater 4:137-147

Cowin SC (1986) Wolff's law of trabecular architecture at remodeling equilibrium. J Biomech Eng 108:83-88

Cowin SC (1997) Remarks on the paper entitled fabric and elastic principal directions of cancellous bone are closely related. J Biomech 30:1191-1192

Darcy H (1856) Les Fontains Publiques de la Ville de Dijon. Dalmont, Paris

Ericksen JL (1960) Tensor fields. In: Truesdell CA (ed) Encyclopedia of physics. Springer, Berlin pp 794-858

Formica CA (1998) Standardization of BMD measurements. Osteoporos Int 8:1-3

Gandolini G, Salvioni PM (2004) Is BMD measurement an adequate surrogate for anti-fracture efficacy?. Aging Clin Exp Res 16:29_ 32 
Glorieux FH, Travers R, Taylor A, Bowen JR, Rauch F, Norman M, Parfitt AM (2000) Normative data for iliac bone histomorphometry in growing children. Bone 26(2):103-109

Grigorian M, Shepherd JA, Cheng XG, Njeh CF, Toschke JO, Genant HK (2002) Does osteoporosis classification using heel BMD agree across manufacturers? Osteoporos Int 13:613-617

Grimm MJ, Williams JL (1997a) Assessment of bone quantity and 'quality' by ultrasound attenuation and velocity in the heel. Clin Biomech (Bristol, Avon) 12:281-285

Grimm MJ, Williams JL (1997b) Measurements of permeability in human calcaneal trabecular bone. J Biomech 30:743-745

Hans D, Fuerst T, Uffmann M (1996) Bone density and quality measurement using ultrasound. Curr Opin Rheumatol 8:370-375

Harrigan T, Mann RW (1984) Characterization of microstructural anisotropy in orthotropic materials using a second rank tensor. J Mat Sci 19:761-769

Hengsberger S, Kulik A, Zysset P (2001) A combined atomic force microscopy and nanoindentation technique to investigate the elastic properties of bone structural units. Eur Cell Mater 1:12-17

Hengsberger S, Kulik A, Zysset P (2002) Nanoindentation discriminates the elastic properties of individual human bone lamellae under dry and physiological conditions. Bone 30:178-184

Hildebrand T, Laib A, Müller R, Dequeker J, Rüegsegger P (1999) Direct three-dimensional morphometric analysis of human cancellous bone: microstructural data from spine, femur, iliac crest, and calcaneus. J Bone Mineral Res 14:1167-1174

Hill R (1952) The elastic behaviour of crystalline aggregate. Proc Phys Soc A 65:349-354

Hilliard JE (1967) Determination of structural anisotropy. In: Stereology-Proceedings of the 2nd International Congress for Stereology, Chicago. Springer, Berlin, p 219

Hoffler CE, Moore KE, Kozloff K, Zysset PK, Goldstein SA (2000) Age, gender, and bone lamellae elastic moduli. J Orthop Res $18: 432-437$

Hoffler CE, Moore KE, Kozloff K, Zysset PK, Brown MB, Goldstein SA (2000) Heterogeneity of bone lamellar-level elastic moduli. Bone 26:603-609

Hosokawa A, Otani T (1997) Ultrasonic wave propagation in bovine cancellous bone. J Acoust Soc Am 101:558-562

Hosokawa A, Otani T (1998) Acoustic anisotropy in bovine cancellous bone. J Acoust Soc Am 103:2718-2722

Johnson DL, Koplik J, Dashen R (1987) Theory of dynamic permeability and tortuosity in fluid-saturated porous media. J Fluid Mech 176:379-402

Jones AC, Sheppard AP, Sok RM, Arns CH, Limaye A, Averdunk H, Brandwood A, Sakellariou A, Senden TJ, Milthorpe BK, Knackstedt MA (2004) Three-dimensional analysis of cortical bone structure using X-ray micro-computed tomography. Physica A: Statistical Mechanics and its Applications 339(1-2):125-130. Proceedings of the International Conference New Materials and Complexity

Jorgensen CS, Kundu T (2002) Measurement of material elastic constants of trabecular bone: a micromechanical analytic study using a $1 \mathrm{GHz}$ acoustic microscope. J Orthop Res 20:151-158

Kanatani K (1983) Characterization of structural anisotropy by fabric tensors and their statistical test. J Jpn Soil Mech Found Eng 23:171

Kanatani K (1984a) Distribution of directional data and fabric tensors. Int J Eng Sci 22:149-164

Kanatani K (1984b) Stereological determination of structural anisotropy. Int J Eng Sci 22:531-546

Kanatani K (1985) Procedures for stereological estimation of structural anisotropy. Int J Eng Sci 23:587-596

Kaptoge S, Benevolenskaya LI, Bhalla AK, Cannata JB, Boonen S, Falch JA, Felsenberg D, Finn JD, Nuti R, Hoszowski K, Lorenc R, Miazgowski T, Jajic I, Lyritis G, Masaryk P, Naves-Diaz M,
Poor G, Reid DM, Scheidt-Nave C, Stepan JJ, Todd CJ, Weber K, Woolf AD, Roy DK, Lunt M, Pye SR, O'neill TW, Silman AJ, Reeve J (2005) Low BMD is less predictive than reported falls for future limb fractures in women across Europe: results from the European prospective osteoporosis study. Bone 36:387-398

Kleerekoper M, Nelson DA (2005) Is BMD testing appropriate for all menopausal women? Int J Fertil Womens Med 50:61-66

Kohles SS, Roberts JB (2002) Linear poroelastic cancellous bone anisotropy: trabecular solid elastic and fluid transport properties. J Biomech Eng 124:521-526

Kohles SS, Roberts JB, Upton ML, Wilson CG, Bonassar LJ, Schlichting AL (2001) Direct perfusion measurements of cancellous bone anisotropic permeability. J Biomech 34:11971202

Li GP, Bronk JT, An KN, Kelly PJ (1987) Permeability of cortical bone of canine tibiae. Microvasc Res 34(3):302-310

Lim TH, Hong JH (2000) Poroelastic properties of bovine vertebral trabecular bone. J Orthop Res 18:671-677

Link TM, Vieth V, Matheis J, Newitt D, Ying L, Rummeny EJ, Majumdar S (2002) Bone structure of the distal radius and the calcaneus vs BMD of the spine and proximal femur in the prediction of osteoporotic spine fractures. Eur Radiol 12:401-408

Mason WP (1958) Physical acoustics and the properties of solids. Van Nostrand Reinhold, Princeton

Matsuura M, Eckstein F, Lochmüller E-M, Zysset PK (2008) The role of fabric in the quasi-static compressive mechanical properties of human trabecular bone from various anatomical locations. Biomech Model Mechanobiol 7:27-42

Morgan EF, Bayraktar HH, Keaveny TM (2003) Trabecular bone modulus-density relationships depend on anatomic site. J Biomech 36:897-904

Nauman EA, Fong KE, Keaveny TM (1999) Dependence of intertrabecular permeability on flow direction and anatomic site. Ann Biomed Eng 27:517-524

Nicholson PH, Cheng XG, Lowet G, Boonen S, Davie MW, Dequeker J, Vander Perre G(1997) Structural and material mechanical properties of human vertebral cancellous bone. Med Eng Phys 19:729-737

Nicholson PHF, Müller R, Lowet G, Cheng XG, Hildebrand T, Rüegsegger P, Vander Perre G, Dequeker J, Boonen S (1998) Do quantitative ultrasound measurements reflect structure independently of density in human vertebral cancellous bone? Bone 23:425-431

Nielsen SP (2000) The fallacy of BMD: a critical review of the diagnostic use of dual X-ray absorptiometry. Clin Rheumatol 19:174-183

Njeh CF, Fuerst T, Diessel E, Genant HK (2001) Is quantitative ultrasound dependent on bone structure? A reflection. Osteoporos Int 12:1-15

Oda M (1976) Fabrics and their effects on the deformation behaviors of sand. Department of Foundation Engineering, Saitama University, Japan

Oda M, Konishi J, Nemat-Nasser S (1980) Some experimentally based fundamental results on the mechanical behavior of granular materials. Geotechnique 30:479

Oda M, Nemat-Nasser S, Konishi J (1985) Stress induced anisotropy in granular masses. Soils Found 25:85

Odgaard A (1997a) Three-dimensional methods for quantification of cancellous bone architecture. Bone 20:315-328

Odgaard A, Kabel J, van Rietbergen B, Dalstra M, Huiskes R (1997b) Fabric and elastic principal directions of cancellous bone are closely related. J Biomech 30:487-495

Odgaard A (2001) Quantification of cancellous bone architecture. In: Cowin SC (ed) Bone mechanics handbook. CRC Press, Boca Raton

Parfitt AM, Mathews CH, Villanueva AR, Kleerekoper M, Frame B, Rao DS (1983) Relationships between surface, volume, and thick- 
ness of iliac trabecular bone in aging and in osteoporosis. Implications for the microanatomic and cellular mechanisms of bone loss. J Clin Invest 72(4):1396-1409

Perrot C, Chevillotte F, Panneton R, Allard J-F, Lafarge D (2008) On the dynamic viscous permeability tensor symmetry. J Acoust Soc Am Express Lett 124:EL210-EL217

Plona TJ, Johnson DL (1983) Acoustic properties of porous systems: I. phenomenological description. In: Johnson DL, Sen PN (eds) Physcis and chemistry of porous media, AIP conference proceedings No. vol. 107, pp 89-104

Rehman MT, Hoyland JA, Denton J, Freemont AJ (1994) Age related histomorphometric changes in bone in normal British men and women. J Clin Pathol 47(6):529-534

Rho JY, Roy ME 2nd, Tsui TY, Pharr GM (1999) Elastic properties of microstructural components of human bone tissue as measured by nanoindentation. J Biomed Mater Res 45:48-54

Rho JY, Tsui TY, Pharr GM (1997) Elastic properties of human cortical and trabecular lamellar bone measured by nanoindentation. Biomaterials 18:1325-1330

Roy ME, Rho JY, Tsui TY, Evans ND, Pharr GM (1999) Mechanical and morphological variation of the human lumbar vertebral cortical and trabecular bone. J Biomed Mater Res 44:191-197

Sakata S, Barkmann R, Lochmuller EM, Heller M, Gluer CC (2004) Assessing bone status beyond BMD: evaluation of bone geometry and porosity by quantitative ultrasound of human finger phalanges. J Bone Miner Res 19:924-930

Satake M (1982) Fabric tensor in granular materials. In: Vermeer PA, Lugar HJ (eds) Deformation and failure of granular materials. Balkema, Rotterdam p 63

Sharma MD (2005) Propagation of inhomogeneous plane waves in dissipative anisotropic poroelastic solids. Geophys J Int 163:981-990

Sharma MD (2008) Propagation of harmonic plane waves in a general anisotropic porous solid. Geophys J Int 172(3):982-994

Siffert R, Kaufman J (2006) Ultrasonic bone assessment: the time has come. Bone 40(1):5

Steiger P (1995a) Standardization of measurements for assessing BMD by DXA. Calcif Tissue Int 57:469
Steiger P (1995b) Standardization of postero-anterior (PA) spine BMD measurements by DXA. Committee for Standards in DXA. Bone 17:435

Thompson M, Willis JR (1991) A reformation of the equations of anisotropic poroelasticity. J Appl Mech 58:612-616

Turner CH, Cowin SC (1987) On the dependence of the elastic constants of an anisotropic porous material upon porosity and fabric. J Mater Sci 22:3178-3184

Turner CH, Cowin SC, Rho JY, Ashman RB, Rice JC (1990) The fabric dependence of the orthotropic elastic properties of cancellous bone. J Biomech 23:549-561

Turner CH, Rho J, Takano Y, Tsui TY, Pharr GM (1999) The elastic properties of trabecular and cortical bone tissues are similar: results from two microscopic measurement techniques. J Biomech $32: 437-441$

Van Rietbergen B, Odgaard A, Kabel J, Huiskes R (1996) Direct mechanical assessment of elastic symmetries and properties of trabecular bone architecture. J Biomech 29:1653-1657

Van Rietbergen B, Odgaard A, Kabel J, Huiskes R (1998) Relationships between bone morphology and bone elastic properties can be accurately quantified using high-resolution computer reconstructions. J Orthop Res 16:23-28

Whitehouse WJ (1974a) The quantitative morphology of anisotropic trabecular bone. J Microsc 101:153-168

Whitehouse WJ, Dyson ED (1974b) Scanning electron microscope studies of trabecular bone in the proximal end of the human femur. J Anat 118:417-444

Williams JL (1992) Ultrasonic wave propagation in cancellous and cortical bone: prediction of some experimental results by Biot's theory. J Acoust Soc Am 91:1106-1112

Yang G, Kabel J, van Rietbergen B, Odgaard A, Huiskes R, Cowin SC (1999) The anisotropic Hooke's law for cancellous bone and wood. J Elast 53:125-146

Zysset PK, Guo XE, Hoffler CE, Moore KE, Goldstein SA (1999) Elastic modulus and hardness of cortical and trabecular bone lamellae measured by nanoindentation in the human femur. J Biomech 32:1005-1012 